\title{
Optical structure and star formation in blue compact dwarf galaxies. \\ I. Observations and profile decomposition
}

\author{
P. Papaderos ${ }^{1}$, H.-H. Loose ${ }^{1,2,4}$, T.X. Thuan ${ }^{3,4}$ and K.J. Fricke ${ }^{1}$ \\ 1 Universitäts-Sternwarte, Geismarlandstraße 11, D-37083 Göttingen, Germany \\ 2 deceased August 5, 1993 \\ 3 Astronomy Department, University of Virginia, P.O. Box 3818, University Station, Charlottesville, VA 22903, U.S.A. \\ 4 Visiting Astronomer at Kitt Peak National Observatory (KPNO), which is operated by AURA, Inc., under contract \\ with the National Science Foundation
}

Received December 18, 1995; accepted April 16, 1996

\begin{abstract}
We present new $B$ and $R$ CCD surface photometry for a sample of 4 Blue Compact Dwarf Galaxies (BCDs) and 2 higher luminosity starburst galaxies. We find that the BCD surface brightness profiles (SBPs) can be generally well fitted by the sum of the light of 3 distinct components: 1) an exponential component at large radii, with or without a central flattening which describes the underlying older stellar population; 2) a plateau component at intermediate radii and 3) a gaussian component at small radii. The last 2 components describe the ongoing starburst superimposed on the older stellar component. We apply the 3-component decomposition scheme to 8 more BCDs from the literature and, for the total sample of 14 galaxies, derive structural properties such as central surface brightness and scale length, and perform a deprojection to obtain the luminosity density distribution for each component. We also derive $B-R$ color profiles and find that BCDs show generally a reddening outwards, followed by a blueing beyond a transition radius.
\end{abstract}

Key words: galaxies: compact; photometry; structure; fundamental parameters

\section{Introduction}

Following the pioneering work of Searle \& Sargent (1972), the main defining characteristics of the class of Blue Compact Dwarf Galaxies (BCDs) were formulated by Thuan \& Martin (1981): low luminosity $\left(M_{B} \gtrsim-18\right)$, compact optical size, and spectra similar to those of Hil regions. Later work showed BCDs to be metal-deficient - $\left(Z_{\odot} / 50 \lesssim Z \lesssim Z_{\odot} / 3\right)$ as compared to the solar vicinity with a mean abundance of $Z_{\odot} / 10$ (Kunth \& Sargent 1986; Thuan et al. 1994). Both their optical spectra which exhibit strong emission lines superimposed on a nearly featureless blue continuum and their blue optical colors imply the presence of a large population of young OB stars. Formation rates of massive $\mathrm{OB}$ stars were estimated to range between 0.1 and $1 M_{\odot} \mathrm{yr}^{-1}$ (Fanelli et al. 1988). The current level of star formation in BCDs cannot be maintained for more than $\sim 10^{9} \mathrm{yr}$ without exhausting their gas reservoir $\left(\sim 10^{8}-10^{9} M_{\odot}\right.$, Thuan \& Martin 1981). Thus, star formation in $\mathrm{BCDs}$ has to proceed in intermittent bursts,

Send offprint requests to: papade@usw008.dnet.gwdg.de lasting less than $10^{7} \mathrm{yr}$ and separated by long ( $1-3 \mathrm{Gyr}$ ) quiescent phases (Thuan 1991).

There have been many theoretical studies of the evolution of the photometric and chemical properties of BCDs (Lequeux et al. 1979, Matteucci \& Chiosi 1983; Vigroux et al. 1987; Krüger et al. 1991; Marconi et al. 1994; Pilyugin 1994; Roy \& Kunth 1995). The main outcome of many of these studies was that the chemical abundances of BCDs cannot be adequately accounted for without invoking galactic winds. Strong galactic winds are expected to be powered by the collective input of energy and momentum into the interstellar medium (ISM) from OB stars and SNe (Vader 1986; De Young \& Gallagher 1990; De Young \& Heckman 1994). Indeed, recent studies have revealed in several BCDs a violently disturbed, turbulent gaseous component with a velocity close to or above the escape velocity (Sargent \& Filippenko 1991; Roy et al. 1992; Izotov et al. 1993; Lequeux et al. 1995). Further observational support for starburst-driven winds in BCDs comes from narrow-band optical imaging and X-ray observations (Meurer et al. 1992; Papaderos et al. 1994; Marlowe et al. 1995). 
The very low metallicity of BCDs has raised the question whether they are genuinely young systems undergoing their first major starburst event. A strong objection against this idea came from deep CCD imaging which revealed a faint extended stellar envelope in the vast majority of them (Loose \& Thuan 1985). Observational work in the optical and NIR (Thuan 1983; Campbell \& Terlevich 1984; Thuan 1985; Kunth et al. 1988; Freitag 1992; James 1994) and evolutionary synthesis models (Krüger 1991, 1995) have shown that this underlying faint component consists of stars older than several Gyr. Loose \& Thuan (1985) subdivided BCDs possessing a faint stellar component into two major classes based on the morphologies of the High Surface Brightness (HSB) starforming region and the Low Surface Brightness (LSB) underlying stellar component. The vast majority of BCDs $(\gtrsim 70 \%$ ) belong to the $\mathrm{iE}$ class, with a smooth elliptical LSB component on which are superimposed several irregular knots of star formation, and $\sim 20 \%$ to the $\mathrm{nE}$ class with nuclear star formation activity superimposed on a LSB elliptical component. Hence, from the morphological point of view the classes $\mathrm{nE}$ and $\mathrm{iE}$ differ only in the degree in which star formation is confined to the center of their elliptical or circular LSB stellar component. A very small fraction of BCDs ( $\lesssim 10 \%)$ does not possess an underlying stellar component.

Despite the extensive work carried out during the past decade, the BCD phenomenon remains puzzling. Three long-standing questions are still unanswered.

i) What is the physical origin of the starburst activity? Since most BCDs $(\gtrsim 70 \%)$ are isolated (Salzer 1989, Campos-Aguilar \& Moles 1991; Rosenberg et al. 1994; Pustil'nik et al. 1995; Telles \& Terlevich 1995; Lindner et al. 1996) their activity is unlikely to be initiated by dynamical interactions or merging with close companions. Most probably, the origin of both bursts of star formation and the long interburst quiescent period is associated with internal processes not yet well understood.

ii) What is the role played by the older LSB stellar component in the global star formation history? This high mass-to-light ratio component of BCDs is mainly responsible for the gravitational potential within which the gas collapses to form new stars. Thus, it is important to determine its structural properties and investigate in which way it may influence the global star formation.

iii) How does a BCD appear in an interburst phase and after its gas content is depleted? Since BCDs experience $\lesssim 10$ bursts during their evolution each lasting for $\lesssim 10^{7}$ yr (Thuan 1991) they spend $\sim 99 \%$ of their lifetime in a dormant state. The understanding of this quiescent phase is also crucial for the development of a coherent evolutionary picture for dwarf galaxies in general. It is commonly thought that dwarf irregulars (dIs) are BCDs in a quiescent phase and that dwarf ellipticals (dEs) are the final evolutionary stage of BCDs (Thuan 1985; Davies
\& Phillipps 1988). The comparison of the structural properties of the underlying LSB component of BCDs with those of dIs and dEs, will be important for establishing evolutionary connections.

In order to address these fundamental questions we obtained broad-band CCD surface photometry of a sample of BCDs. Section 2 describes the observations and data reduction. In Sects. 3 and 4 we discuss the decomposition of the surface brightness profiles into three distinct components: two of them describe the light from the younger stellar population and the third one the old LSB component. In Sect. 5 we derive the intrinsic luminosity densities for each of the components which can be used to constrain the radial mass density distribution and the form of the gravitational potential in BCDs. In Sect. 6 we discuss the color profiles and in Sect. 7 we summarize our results. In an accompanying article (Paper II), we shall investigate relationships between the properties of the old underlying stellar component and those of the superimposed young starburst component. Furthermore, the structural properties of the old component will be compared to those of other types of dwarf galaxies in order to check for evolutionary connections. We adopt throughout a Hubble constant of $H_{0}=75 \mathrm{~km} \mathrm{~s}^{-1} \mathrm{Mpc}^{-1}$.

\subsection{Observations}

The data were obtained with the Kitt Peak National Observatory (KPNO) No. $10.9 \mathrm{~m}$ telescope during the period 1983 May 15-17. The CCD camera consisting of a RCA $320 \times 512$ pixels chip, was used at the $f / 7.5$ focus of the telescope giving a scale of $0 ! 86 / \mathrm{pixel}$ and a field of view of $4^{\prime} 6 \times 7 ! 3$, large enough to allow a precise sky background determination. Images were obtained in two bandpasses in the Mould filter system $\left[B\left(\lambda_{\mathrm{c}}=4400 \AA, \Delta \lambda=1125 \AA\right)\right.$ and $R\left(\lambda_{\mathrm{c}}=6500 \AA, \Delta \lambda=1280 \AA\right)$ ], close to the Johnson system. Calibration exposures taken each night consisted of bias frames, dark frames, and flat fields. The latter were made by exposing the CCD to a patch illuminated by a spotlight on the inside of the dome. Throughout the observations the weather was photometric. There were optical problems with the telescope which made the images of stellar objects in the $B$ and $R$ frames not perfectly round. The stellar point spread function, $\mathrm{PSF} \approx \exp \left[-\frac{1}{2}(r / \sigma)^{2}\right]$, as determined from four separate stars, gave $\sigma_{B}=22^{\prime \prime} 6$ and $\sigma_{R}=2 ! 2$. Thus the inner parts of the SBPs $\left(R^{*} \lesssim 5^{\prime \prime}\right)$ which are affected by the above problems may not reproduce precisely the luminosity and color distributions of the most luminous components in the observed targets. Usual reduction procedures (bias, dark and flat field corrections) were applied to the data (see also Loose \& Thuan 1986), and absolute calibration was accomplished using standard stars from the M 92 photometric calibration field (Christian et al. 1985) and the blue star Feige 34 (Sargent \& Searle 1968). 
The observed sample consists of six galaxies, chosen from the BCD list of Thuan \& Martin (1981), with the exception of Mkn 297. Although Mkn 297 and I Zw 89 are brighter than $M_{B}=-18$, the magnitude cut-off of Thuan $\&$ Martin (1981), they were included for comparison. We summarize the main properties of the observed galaxies in Table 1.

\subsection{Surface photometry}

\subsubsection{Derivation of surface brightness profiles}

The derivation of a surface brightness profile (SBP) is equivalent to a transformation of an arbitrary twodimensional flux pattern into a spherically-symmetric intensity distribution $\mu\left(R^{*}\right)$, which is monotonically decreasing with radius $R^{*}$. Here, $R^{*}$ is the photometric radius corresponding to the surface brightness level $\mu$. The most simple technique for obtaining a SBP is to sum up the flux enclosed within a circular aperture for a sequence of aperture sizes. Alternatively, for elliptical galaxies, one may use instead of rings, a set of elliptical apertures adjusted to the position angle and ellipticity of the galaxy and centered on it. Such a technique is not appropriate for BCDs mainly because they contain off-center irregular and clumpy HSB star-forming regions and their isophotes frequently show a strong twist with decreasing intensity.

Because of the large variety of isophote shapes in BCDs, we applied three distinct methods for different ranges of intensity levels. The photometric radius $R^{*}$ at the intensity level $I_{0}$ has been calculated as the equivalent radius of the enclosed area $A\left(I_{0}\right), R^{*}=\sqrt{A\left(I_{0}\right) / \pi}$. The advantage of averaging over an isophotal contour as compared to a simple slice through the luminosity pattern of the galaxy is the increased number $N$ of pixels defining the isophote, and hence the reduction of the uncertainty at $R^{*}$ by a factor $\sqrt{N}$. Contamination of an isophote by foreground stars or background galaxies may in some cases cause a slight overestimation of $A\left(I_{0}\right)$. To avoid such problems, we developed a software package where contaminating objects are interactively determined and rejected. At flux levels below $\sim 4 \times$ (rms noise) we smoothed the CCD images with a $3 \times 3$ pixel median filter to improve the $S / N$, and better define the isophote's shape. Three equivalent methods were employed:

i) At low intensity levels, an improved version of the algorithm described by Loose \& Thuan (1986) was used to fit ellipses to the isophotes. The five parameters necessary to define an ellipse in the plane, the major and minor axes $a$ and $b$, the center coordinates $x_{\mathrm{c}}$ and $y_{\mathrm{c}}$, and the position angle $\phi$, are obtained from a polynomial fit to the isophote by a coordinate transformation. The photometric radius corresponding to the area of the ellipse is $\sqrt{a b}$.

ii) The isophotes of BCDs at intermediate intensity levels are frequently irregular. Their enclosed area can be directly computed by a line integral of the form:

$$
A\left(I_{0}\right)=\frac{1}{2} \oint(x \mathrm{~d} y-y \mathrm{~d} x)
$$

where the integral is evaluated along the isophote. The center of gravity $x_{\mathrm{c}}, y_{\mathrm{c}}$ is defined by

$$
x_{\mathrm{c}}=\frac{1}{3 A} \oint_{I_{0}} x(x \mathrm{~d} y-y \mathrm{~d} x) \quad y_{\mathrm{c}}=\frac{1}{3 A} \oint_{I_{0}} y(x \mathrm{~d} y-y \mathrm{~d} x)
$$

The integration can be performed in both cartesian and polar coordinates. This method yields reliable results when applied to an isophote of arbitrary shape, provided it contains a sufficient number of pixels $(\gtrsim 20)$ and the noise is not dominant.

iii) The central region of $\mathrm{iE}$ BCDs, may consist of several HSB star-forming knots. The area evaluation of every single knot by techniques i) and ii) is time-consuming and may be inaccurate since the isophote contains generally a low number of pixels. In such cases we defined interactively on the monitor screen a polygon of suitable shape within which all pixels of intensity larger than or equal to $I_{0}$ were summed up. The corresponding area is:

$$
A\left(I_{0}\right)=\sum_{i} A_{i}
$$

where $A_{i}$ is the pixel area and $i$ runs over all pixels with $I(x, y) \geq I_{0}$. Tests showed that this method gave reliable results in the situations described above. By contrast, in the outer regions of $\mathrm{BCDs}$ this technique results in a slight overestimation of $R^{*}$ because of noise. Although we have checked that all three techniques gave results in good agreement with each other, we have adopted throughout the results of the method which is most appropriate to the shape of a given isophote.

The uncertainty $\sigma_{\mu}$ in mag of the SBPs at a surface brightness level $\mu$ were estimated assuming that Poisson statistics apply and taking into account inaccuracies in the determination of the background level, as:

$$
\sigma_{\mu}^{2}=\left(\frac{2.5}{I_{0} \ln 10}\right)^{2}\left(\frac{g I}{N}+\delta_{\mathrm{BG}}^{2}\right)+\sigma_{\mathrm{c}}^{2}
$$

where: $I_{0}$ is the number of galaxy counts above the background received per pixel, $I=I_{0}+I_{\mathrm{BG}}$ the sum of the galaxy counts $I_{0}$ and the background counts $I_{\mathrm{BG}}, g(\sim 0.1$ for the KPNO CCD) the conversion factor from the number $n_{\mathrm{e}^{-}}$of detected electrons in one pixel into counts, $\delta_{\mathrm{BG}}$ the uncertainty in the determination of the background in counts, and $N$ the number of pixels defining the isophote. The uncertainties in the calibration $\sigma_{\mathrm{c}}$, not shown in Figs. 1-6, are $0.036 \mathrm{mag}$ in $B$ and $0.01 \mathrm{mag}$ in $R$. The main systematic uncertainties in the SBPs come from the uncertainties in the sky level determination. Our photometry is better than $0.1 \mathrm{mag}$ for surface brightness levels brighter than $23 \mathrm{mag} \operatorname{arcsec}^{-2}$ in $B$ and $22.5 \mathrm{mag} \operatorname{arcsec}^{-2}$ in $R$, 
Table 1. Observed galaxy sample

\begin{tabular}{|c|c|c|c|c|c|c|c|c|}
\hline Object & other designations & $\begin{array}{l}\alpha_{1950} \\
\delta_{1950}\end{array}$ & $\begin{array}{l}l^{\mathrm{II}} \\
b^{\mathrm{II}}\end{array}$ & Filter & $\begin{array}{c}M(\mathrm{HI})^{a} \\
M_{\mathrm{T}}^{a}\end{array}$ & $\begin{array}{l}\Delta t_{\text {obs }} \\
{[\mathrm{min}]}\end{array}$ & $\begin{array}{c}D[\mathrm{Mpc}] \\
{\left[\mathrm{pc} \operatorname{arcscec}^{-1}\right]}\end{array}$ & $\begin{array}{c}m_{B} \\
\text { source }^{b}\end{array}$ \\
\hline (1) & $(2)$ & (3) & (4) & (5) & (6) & (7) & (8) & (9) \\
\hline \multirow[t]{2}{*}{ Haro 3} & Mkn 35, NGC 3353 & 104215.8 & 152.31 & $\mathrm{~B}$ & 0.54 & 60 & 13.9 & 13.15 \\
\hline & UGC 5860 & +561326 & 53.37 & $\mathrm{R}$ & 1.2 & 60 & 67.39 & $(1)$ \\
\hline \multirow[t]{2}{*}{ Mkn 86} & VV 138, Arp 6 & 080942.6 & 173.81 & $\mathrm{~B}$ & 0.24 & 60 & 7.0 & 12.55 \\
\hline & NGC 2537 & +460832 & 32.96 & $\mathrm{R}$ & 0.58 & 60 & 33.9 & $(1)$ \\
\hline \multirow[t]{2}{*}{ Mkn 209} & Haro 29, I Zw 36 & 122350.5 & 134.15 & B & 0.041 & 60 & 4.6 & 14.84 \\
\hline & UGCA 281 & +484613 & 68.08 & $\mathrm{R}$ & 0.18 & 60 & 22.3 & $(1),(2)$ \\
\hline \multirow[t]{2}{*}{ Mkn 297} & NGC 6052, Arp 209 & 160301.4 & 35.31 & B & 11.8 & 50 & 63 & 15.0 \\
\hline & VV 86, UGC 10182 & +204037 & 45.47 & $\mathrm{R}$ & - & 60 & 305 & $(3)$ \\
\hline \multirow[t]{2}{*}{ I Zw 89} & NGC 5633 & 142536.4 & 84.34 & $\mathrm{~B}$ & 2.60 & 60 & 32.4 & 12.96 \\
\hline & UGC 9271 & +462214 & 63.13 & $\mathrm{R}$ & 35.0 & 60 & 158 & (1) \\
\hline \multirow[t]{2}{*}{ I Zw 115} & UGC 9893 & 153119.6 & 75.20 & $\mathrm{~B}$ & 0.13 & 60 & 10.5 & 15.20 \\
\hline & VV 720 & +463709 & 52.90 & $\mathrm{R}$ & 0.15 & 60 & 50.9 & $(1)$ \\
\hline
\end{tabular}

(a): Neutral hydrogen mass $M(\mathrm{HI})$ and total mass $M_{\mathrm{T}}$ (Col. 6) in units of $10^{9} M_{\odot}$.

(b): (1) Thuan \& Martin (1981); (2) Staveley-Smith et al. (1992); (3) Gordon \& Gottesmann (1981).

whereas the errors increase to several tenths of magnitude at $\sim 25.5 \mathrm{mag} \operatorname{arcsec}^{-2}$ in $B$ and $R$, when the light intensity becomes comparable to the background noise.

\subsubsection{Profile fitting}

The derived surface brightness profiles are shown over three radial coordinates to probe different fitting laws. In all cases, a straight line corresponds to a perfect fit to one of the three fitting laws:

i) an exponential law of the form $I=I_{0} \exp \left(-R^{*} / \alpha\right)$ where $I_{0}$ is the central intensity and $\alpha$ the exponential scale length (Figs. 1b-6b). This law is known to provide a good approximation to the disk component of spiral galaxies (Freeman 1970) and to the SBPs of low-luminosity dwarf ellipticals dEs and nucleated dwarf ellipticals dE, Ns (Binggeli et al. 1984; Binggeli \& Cameron 1991).

ii) a power law distribution $\log \left(I / I_{0}\right)=-\tau \log R^{*}$ (Figs. 1c-6c). A power index $\tau \simeq 2$ would correspond to the law proposed by Reynolds (1913) and Hubble (1930) to reproduce the SBPs of giant $\mathrm{E}$ galaxies: $I=$ $I_{0} /\left(1+R^{*} / R_{\mathrm{H}}\right)^{2}$ for $R^{*} \gg R_{\mathrm{H}}$. The Hubble radius $R_{\mathrm{H}}$ is typically $\sim 0.1$ of the effective radius $R_{\mathrm{e}}$ containing half of the total luminosity of the system. Typical power-law indexes in E galaxies range between 1.7 and 2 (Kormendy 1977). From a theoretical point of view, a spherical system in thermodynamic equilibrium would produce an isothermal polytrope distribution $(\tau=1)$ at large radii. A truncated isothermal polytrope distribution, such as the King model (King 1966) has been found to reproduce well the SBPs of low-luminosity dwarf galaxies
(Binggeli et al. 1984; Binggeli \& Cameron 1991; Vader \& Chaboyer 1994).

iii) a de Vaucouleurs law $\log \left(I / I_{\mathrm{e}}\right)=-3.33$ $\left[\left(R^{*} / R_{\mathrm{e}}\right)^{1 / 4}-1\right]$ where $I_{\mathrm{e}}$ is the intensity at the effective radius $R_{\mathrm{e}}$ (Figs. $1 \mathrm{~d}-6 \mathrm{~d}$ ). This fitting law is known to provide a good fit to SBPs of giant Es and of bulges of S0 galaxies.

Except for Mkn 86, with its outer isophotes being nearly circular and Mkn 297 showing an abrupt discontinuity at a surface brightness $\mu_{B} \simeq 21 \mathrm{mag} \operatorname{arcsec}^{-2}$, the position angle $\phi$ varies slowly with radius, typically $\left|\Delta \phi / \Delta R^{*}\right| \sim 1-2$ degree $\operatorname{arcsec}^{-1}$ (Figs. 1f and Figs. 3f-6f). At large radii $\phi$ becomes roughly constant for all galaxies in our sample, similar to the results obtained for BCDs by Diehl (1988), Kunth et al. (1988) and Meurer et al. (1994).

\subsection{Sample description}

We now describe briefly the observed galaxies:

i) Haro 3 (Fig. 1) is a BCD of iE type. A contour plot of the $\mathrm{B}$ frame is shown in Fig. 1a and Fig. 1f illustrates the characteristic isophotal twist as function of radius. In the color map of Haro 3 there is a sequence of three successive knots aligned from northeast to southwest with increasing blue colors. Kunth et al. (1988) reported in the BCDs Tol 2 and Tol 3 a similar sequence of blue knots, interpreting that as a signature of propagating star-formation. The SBP of Haro 3 in B can be described best by a $R^{1 / 4}$ fitting law.

ii) Mkn 86 (Fig. 2) is an extreme example of an $\mathrm{iE} \mathrm{BCD,}$ exhibiting numerous star-forming knots, the bluest of them having $B-R \sim 0.7$. A striking feature in the SBPs of 

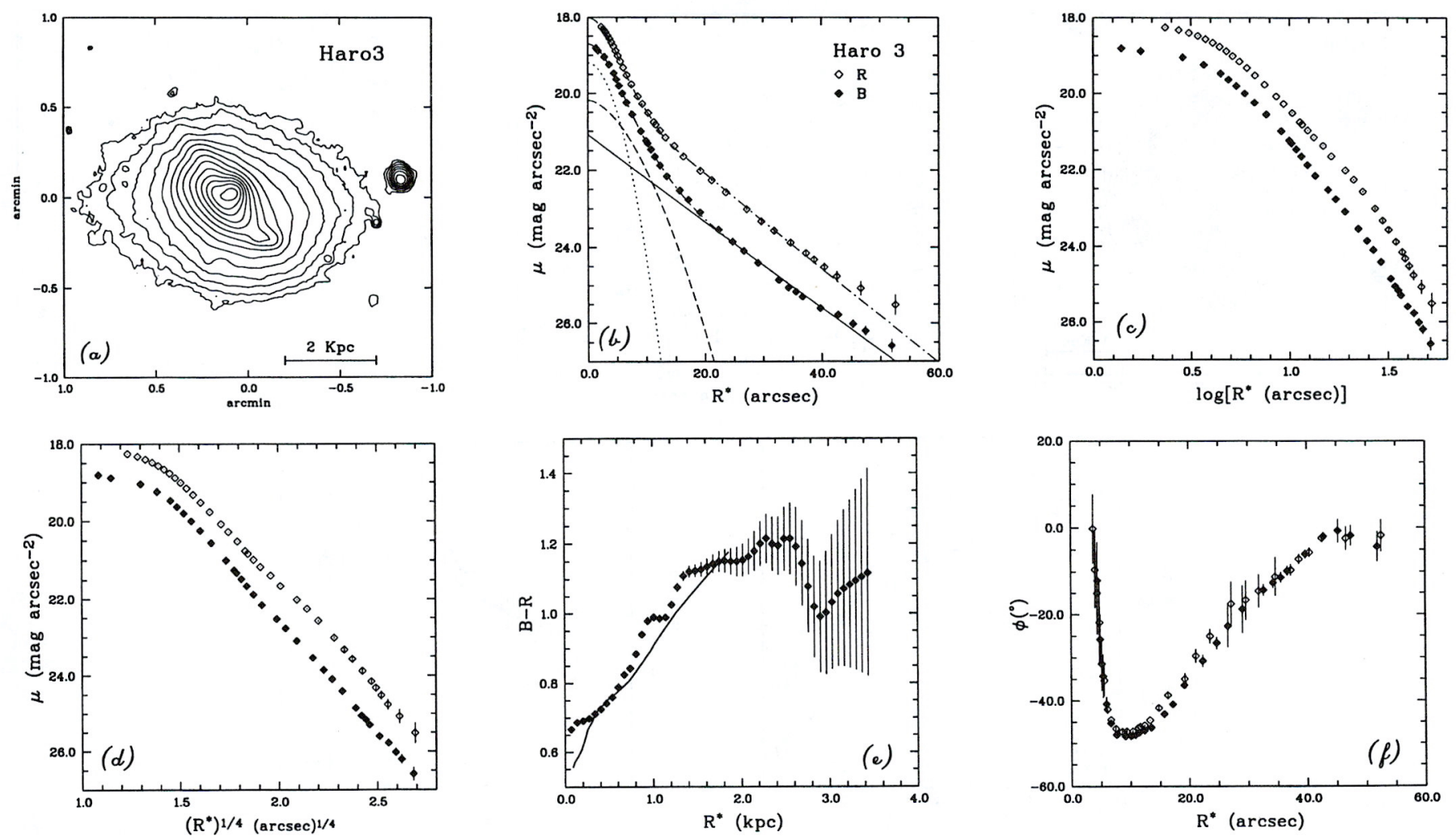

Fig. 1. Haro 3: a) contour plot in $B$, from 19.0 to $25.5 \mathrm{mag} \operatorname{arcsec}^{-2}$ in steps of 0.5 mag. b) linear representation of the SBP in the $B$ and $R$. The exponential, plateau and gaussian luminosity components as obtained by fitting Eq. (9) to the B SBP are shown by a solid, dashed and dotted curve, respectively. c-d) logarithmic and $R^{* 1 / 4}$ representation of the SBPs in $B$ and $R$, e) $B-R$ profile as derived with method I and II (Sect.6), f) position angle $\phi$ vs. $R^{*}$

this BCD is a plateau (nearly constant intensity as a function of $R^{*}$ ) at intermediate intensity levels reminiscent of the bulge component in SBPs of S0 galaxies. Contrary to Haro 3, none of the fitting laws shown in Figs. 2b-d can satisfactorily describe the observed intensity distribution. iii) The iE BCD Mkn 209 (Fig. 3) is the most compact in our sample with an optical radius of $\sim 0.6 \mathrm{kpc}$ at $25 \mathrm{mag} \operatorname{arcsec}^{-2}$. Fanelli et al. (1988) concluded from synthesis analysis of UV spectra that this system may be experiencing currently only its first or second starburst event. A less prominent clump $\sim 18^{\prime \prime}$ eastwards from the brightest knot (Fig. 2a) does suggest a previous episode of star formation. Recent HST observations (Deharveng et al. 1994) of the central $11^{\prime \prime} \times 11^{\prime \prime}$ region uncovered a massive stellar population, consistent with the very blue colors $(B-R \sim 0.0)$ we derived here. This galaxy is of particular interest because it has been discussed as a possible example of a BCD whose mass is dominated by dark matter (Viallefond \& Thuan 1983).

iv) Although the morphology of Mkn 297 (Fig. 4) resembles that of a BCD of type iE, its luminosity $\left(M_{B}=\right.$ $-20.4)$ is brighter than the luminosity cutoff of $M_{\mathrm{B}}=-18$ (Thuan \& Martin 1981) and it is not a bona-fide dwarf galaxy. Hecquet et al. (1987) have resolved the central re- gion in $\sim 40$ knots, one of them coinciding with the strong radio source Mrk 297A (Heeschen et al. 1983). Spectroscopic observations (Alloin \& Dufflot 1979) revealed two kinematically decoupled components, giving circumstantial evidence for the interpretation that Mkn 297 is a dynamically perturbed system (Taniguchi \& Noguchi 1991). Its $B-R$ colors increase inwards which suggests a high internal extinction near the central region. This may result from large amounts of gas driven inwards, a process known to occur in interacting galaxies (Barnes \& Hernquist 1991).

v) The starburst system IZw 89 (Fig. 5) has been included in the Thuan \& Martin (1981) sample because of its blue $U B V$ colors, although it is clearly a nondwarf $\left(M_{B}=-20.2\right.$, Thuan \& Martin 1981) spiral galaxy (Fig. 5a). The knots of star-formation are off-center, probably associated with spiral arms. The SBPs of I Zw 89 show an exponential intensity decrease for intermediate radii $10^{\prime \prime} \lesssim R^{*} \lesssim 30^{\prime \prime}$, and a flattening for $R^{*} \lesssim 10^{\prime \prime}$. As for Mkn 297, I Zw 89 has been included in our sample for comparison.

vi) The iE BCD IZw 115 (Fig. 6) was described by Vorontsov - Velyaminov (1977) as "a pair of coalescents". An interesting feature of this $\mathrm{BCD}$ is its asymmetric 

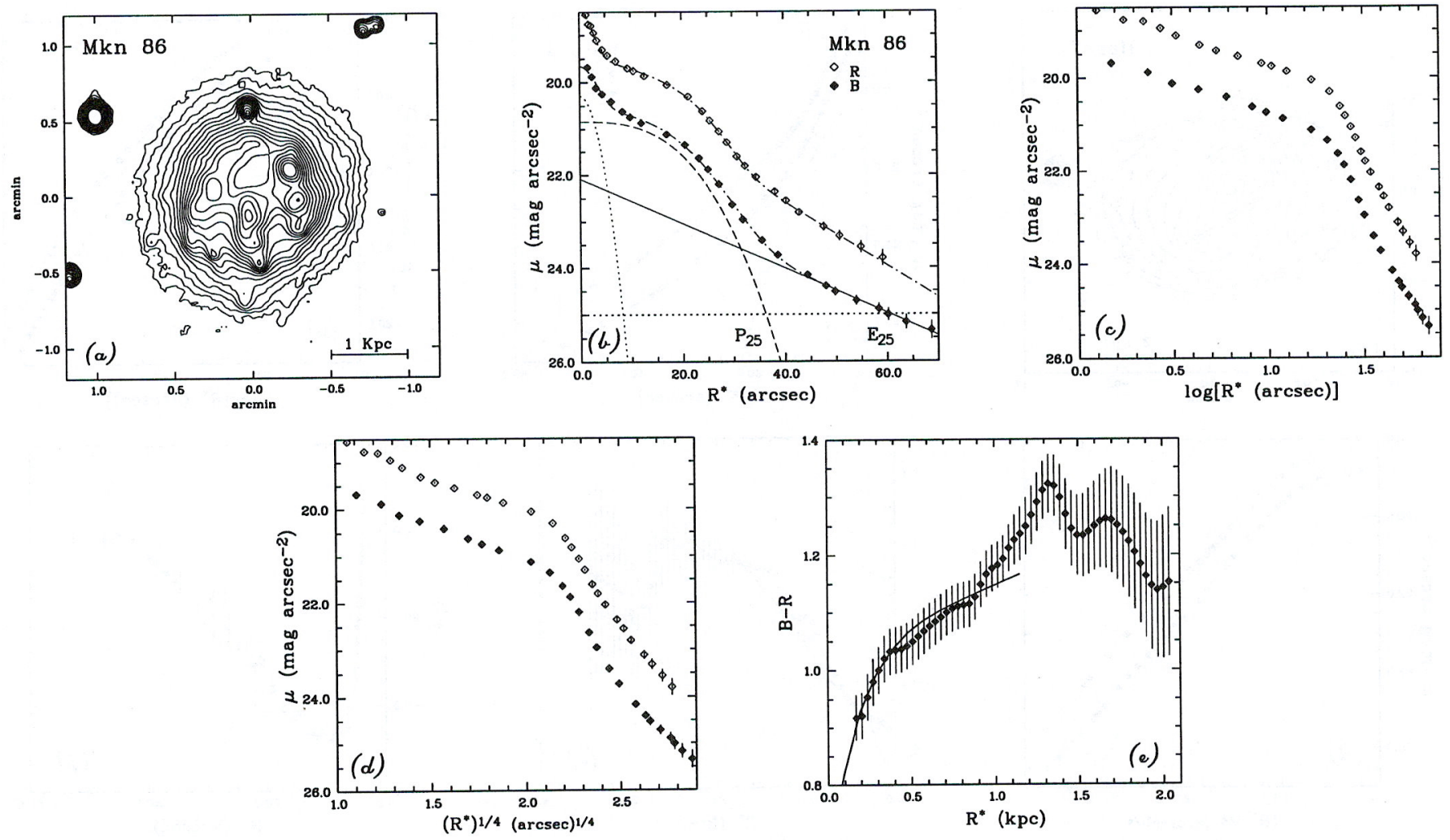

Fig. 2. Mkn 86: a) contour plot in $\mathrm{B}$, from 20.25 to $24.5 \mathrm{mag} \operatorname{arcsec}^{-2}$ in steps of $0.25 \mathrm{mag}$. b) linear representation of the SBP in the $B$ and $R$. The exponential, plateau and gaussian luminosity components as obtained by fitting Eq. (9) to the B SBP are shown by a solid, dashed and dotted curve, respectively. The extents of the starburst component $P_{25}$ and of the LSB component $E_{25}$ measured at $25 \mathrm{mag} \operatorname{arcsec}^{-2}$ are indicated. c-d) linear, logarithmic and $R^{* 1 / 4}$ representation of the SBP in $B$ and $R$ and e) $B-R$ profile as derived with method I and II (Sect. 6)

intensity distribution along the major axis, caused by an abrupt drop in surface brightness southwest from its optical center. IZw 115 is the only member of the sample under study which exhibits boxy-like outer isophotes, possibly the signature of a dynamically young, unrelaxed underlying stellar component. This is in accord with the blue colors of the outer parts of its color profile (Fig. 6e). In the SBPs of $\mathrm{IZw} 115$ the plateau feature at intermediate radii is only marginally present.

\subsubsection{Characteristics of the surface brightness profiles}

None of the fitting laws described above can satisfactorily reproduce the derived SBPs over their entire intensity range. While the exponential law fits well the outer parts of the SBPs, it falls below of them at intermediate intensity levels. In most cases, this is due to the presence of a pronounced plateau feature. This is most obvious for Mkn 86, where for $\mu_{B} \lesssim 24 \mathrm{mag} \operatorname{arcsec}^{-2}$ the exponential fitting law falls below the SBP which is also the case for Mkn 209 and Mkn 297 for $\mu_{B} \lesssim 25 \mathrm{mag} \operatorname{arcsec}^{-2}$. Diehl (1988) has also reported a number of BCDs with SBPs well fitted by an exponential law at low surface brightness levels, while exhibiting a marked plateau at intermediate surface brightness levels (Mkn 5, Mkn 36, Mkn 49, Mkn 400 and Mkn 487; see Fig. 7). Other examples are VCC 213, VCC 562 and VCC 1179 in the Virgo Cluster (Binggeli \& Cameron 1993), RMB 56 (Drinkwater \& Hardy 1991), RMB 46 (Bothun et al. 1986), Tol 2 (Kunth et al. 1988) and a number of Hir galaxies in the Wasilewski sample studied by Bothun et al. (1989).

For I Zw 115, an exponential law gives an acceptable fit to the data for nearly all radii, although slightly overestimating the central surface brightness because of a central flattening of the SBP. Such a central flattening is quite usual in $\mathrm{dE}$ galaxies fainter than $-16 \mathrm{mag}$. Profiles exhibiting this kind of behavior have been called type V profiles by Binggeli \& Cameron (1991). Other BCDs with exponential profile flattening at the center are RMB 175 and Bo 146 (Drinkwater \& Hardy 1991). In both latter BCDs, however, the SBPs steepen again at radii close to the resolution limit, producing a spike-like central excess. Such profiles bear a qualitative resemblance to those of nucleated dwarf elliptical galaxies (dE,N; Binggeli \& Cameron 1991) and numerous LSB galaxies (Bothun et al. 1991; McGaugh \& Bothun 1994; Rönnback \& Bergvall 1994). 

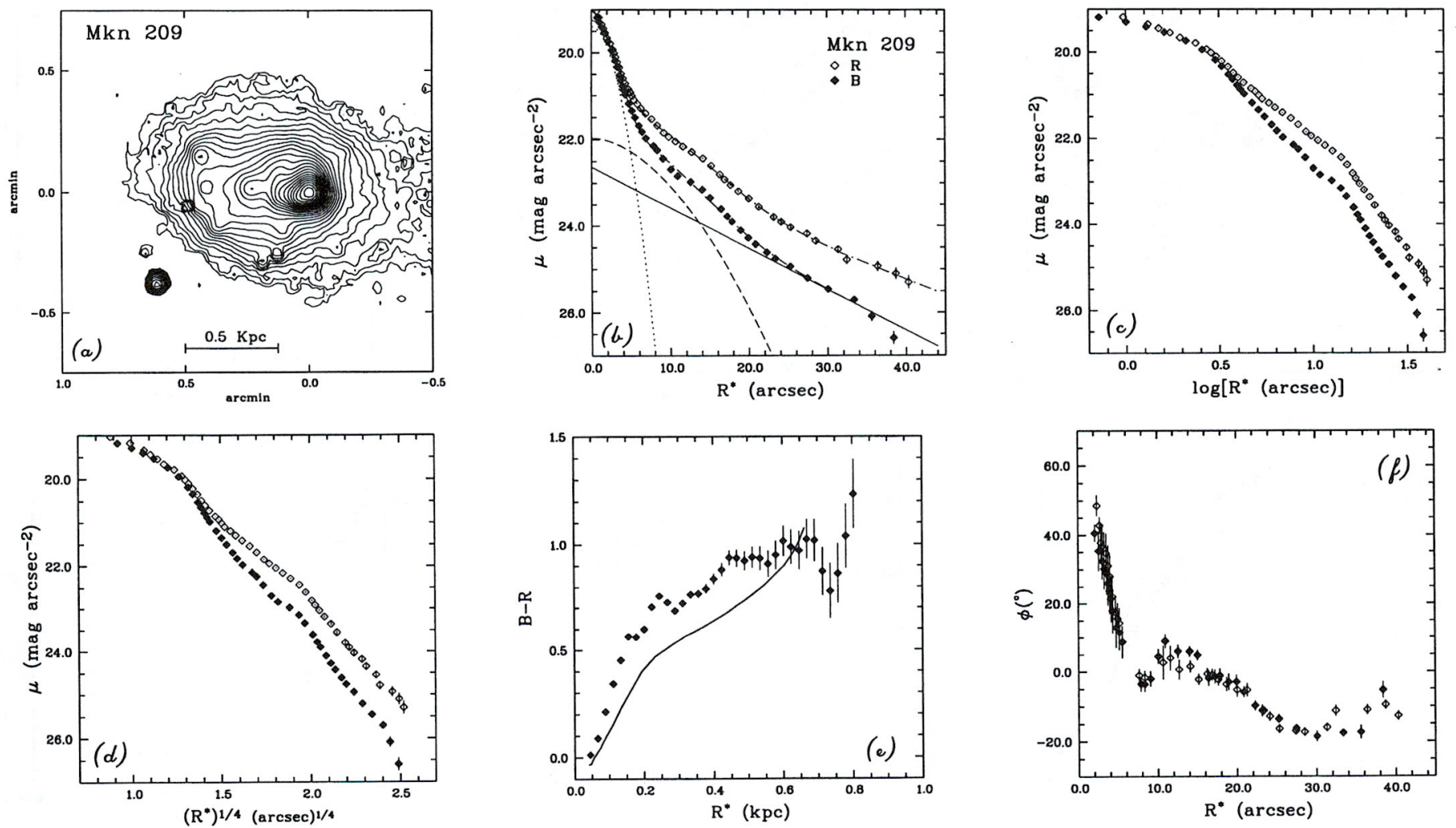

Fig. 3. Mkn 209: a) contour plot in $B$, from 19.5 to $25.5 \mathrm{mag} \operatorname{arcsec}^{-2}$ in steps of 0.25 mag. b) linear representation of the SBP in the $B$ and $R$. The exponential, plateau and gaussian luminosity components as obtained by fitting Eq. (9) to the B SBP are shown by a solid, dashed and dotted curve, respectively. c-d) logarithmic and $R^{* 1 / 4}$ representation of the SBP in $B$ and $R$, e) $B-R$ profile as derived with method I and II (Sect. 6). f) position angle $\phi$ vs. $R^{*}$

An analogous central light depression as compared to a perfect exponential law is also apparent in the SBP of I Zw 89 for $R^{*} \lesssim 10^{\prime \prime}$, which resembles, especially in $R$, the type IIIa profile described by Binggeli \& Cameron (1991).

The de Vaucouleurs law fits best the SBP of Haro 3 in the $B$ band. Other BCDs like Tol 3 (Kunth et al. 1988) and Haro 2 (Loose \& Thuan 1986) belonging to the $\mathrm{nE}$ class have SBPs close to a $R^{1 / 4}$ law while the plateau feature is virtually present. However, even this class does not seem to be entirely uniform regarding the SBPs in different broad-band colors. For example, Haro 2 can be better described by a $R^{1 / 4}$ law than by a power law in the $R$ band, while the situation is reversed in the $B$ band (Loose \& Thuan 1986).

Aside from a few cases, as cited below, a power-law generally fails to reproduce SBPs of BCDs satisfactorily. We note however that the power law index $\tau$, formally derived for the BCDs in our sample and objects in the literature is typically $\gtrsim 2$, in disagreement to the isothermal polytropic distribution (cf. Kunth et al. 1988), and slightly steeper than the power law index $(\$ 2)$ of gE galaxies.

From the limited existing data, we conclude that BCDs show a great variety in their morphology and surface brightness distributions, justifying the attribute of "mixed bag" ascribed to them by Kunth et al. (1988). On the other hand, there appears to exist a basic correspondence between the morphology and the radial intensity distribution. SBPs of BCDs of the $\mathrm{iE}$ type are usually characterized by a plateau at intermediate intensity levels and an exponential intensity decrease at low surface brightness levels. By contrast, SBPs of BCDs of the $\mathrm{nE}$ type, with a more regular morphology in their star-forming component do not exhibit a prominent plateau and can in some cases be well described by a $R^{1 / 4}$ or a power-law over large intensity ranges. In summary, the variety of SBPs of BCDs of the different morphological classes cannot be described by a unique single fitting law over their whole radius range. In the next section we interpret the surface brightness profiles of BCDs in terms of a superposition of 3 distinct luminosity components.

\section{Decomposition of the surface brightness profiles of BCDs}

BCDs are thought to consist of two major stellar constituents: an old LSB stellar population and a superimposed younger stellar population formed in recent starbursts (Searle et al. 1973; Thuan 1985; Loose \& Thuan 

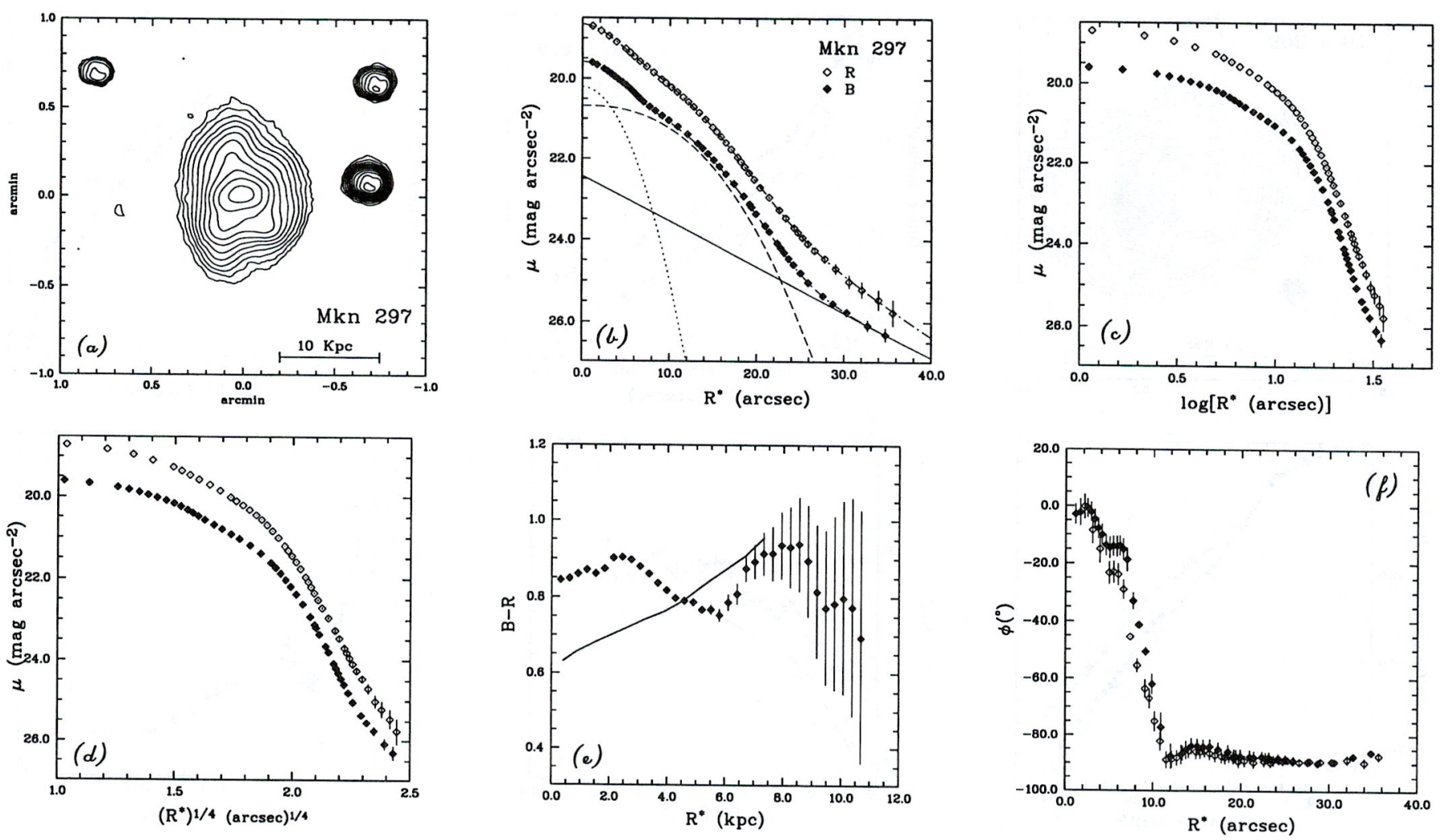

Fig. 4. Mkn 297: a) contour plot in $B$, from 20.0 to $25.0 \mathrm{mag} \operatorname{arcsec}^{-2}$ in steps of 0.5 mag. b) linear representation of the SBP in the $B$ and $R$. The exponential, plateau and gaussian luminosity components as obtained by fitting Eq. (9) to the B SBP are shown by a solid, dashed and dotted curve, respectively. c-d) linear, logarithmic and $R^{* 1 / 4}$ representation of the SBP in $B$ and $R, \mathbf{e )} B-R$ profile as derived with method I and II (Sect. 6). f) position angle $\phi$ vs. $R^{*}$

1985). A quantitative study of the structure and luminosity of these stellar populations can be achieved by an appropriate decomposition of the surface brightness profiles. Kunth et al. (1988) performed a decomposition of the SBPs of Tol 2, Tol 3 and I Zw 123 (Mkn 487) into a disc and bulge component described by an exponential and a $R^{1 / 4}$ law, respectively. Such a two-component model approximates well SBPs of $\mathrm{nE}$ BCDs but fails to reproduce the intermediate plateau component in $\mathrm{iE} \mathrm{BCDs.} \mathrm{We} \mathrm{there-}$ fore use a more general 3-component scheme, initially discussed by Diehl (1988), to describe the SBPs of the BCDs derived in this paper and those taken from the literature.

\subsection{Decomposition scheme}

i) The first component describes the underlying extended dwarf galaxy which contains stars of higher mass-to-light ratio, and which determines to a large degree the gravitational potential in which the gas accumulates and collapses, leading to the formation of the lower $\mathcal{M} / \mathcal{L}$ starburst component. This first component is only partly accessible to direct observations as its light is contaminated by the luminous superimposed starburst component. We can fit its outer, low surface brightness region, which is not polluted by the light from young stellar clusters or nebular emission from ionized gas, by an exponential law of the form:

$$
I_{\mathrm{E}}=I_{\mathrm{E}, 0} \exp \left[-\left(R^{*} / \alpha\right)\right]
$$

or equivalently

$$
\mu \equiv-2.5 \log I_{\mathrm{E}}+\text { constant }=\mu_{\mathrm{E}, 0}+1.086\left(R^{*} / \alpha\right)
$$

where $I_{\mathrm{E}, 0}$ and $\mu_{\mathrm{E}, 0}$ denote respectively the central intensity and the central surface brightness, and $\alpha$ is the exponential scale length. Equation (3) assumes that the exponential law can be extrapolated all the way to $R^{*}=0$. This hypothesis is inappropriate for the BCDs which exhibit an intensity flattening near the center (see Sect. 2.3.1). To take into account these cases, we shall introduce a slightly modified version of Eq. (3) in Sect. 5 (Eq. 22).

ii) The second component characterizes the plateau, seen mainly in SBPs of $\mathrm{iE} \mathrm{BCDs.} \mathrm{It} \mathrm{is} \mathrm{well} \mathrm{fitted} \mathrm{by} \mathrm{a}$ generalized exponential distribution of the form:

$$
I_{\mathrm{P}}=I_{\mathrm{P}, 0} \exp \left[-\left(R^{*} / \beta\right)^{\eta}\right]
$$

or equivalently:

$$
\mu_{\mathrm{P}}=\mu_{\mathrm{P}, 0}+1.086\left[\left(R^{*} / \beta\right)^{\eta}\right]
$$



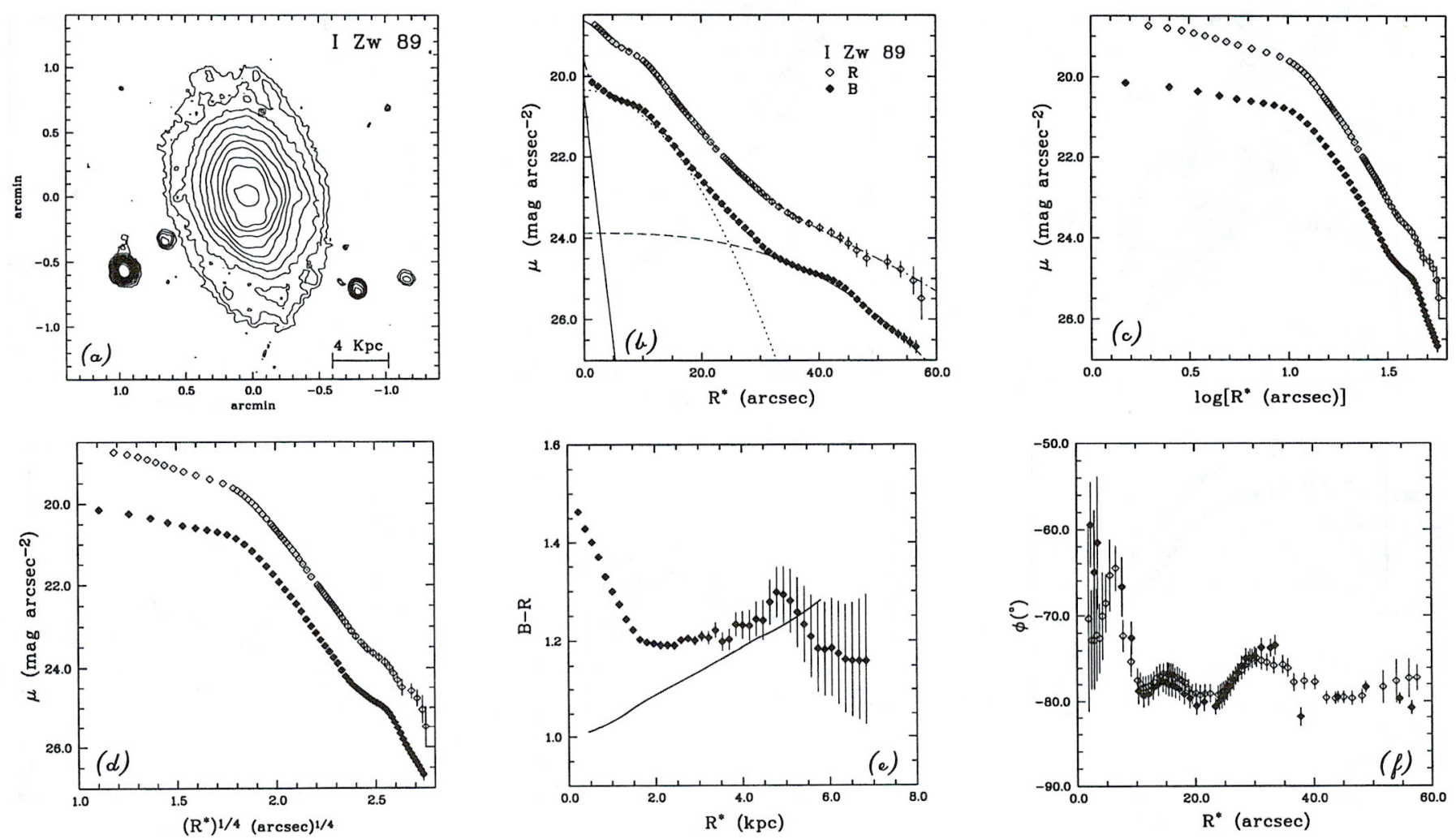

Fig. 5. I Zw 89: a) contour plot in $B$, from 20.5 to $25.5 \mathrm{mag} \operatorname{arcsec}^{-2}$ in steps of $0.5 \mathrm{mag}$. b) linear representation of the SBP in the $B$ and $R$. The exponential, plateau and gaussian luminosity components as obtained by fitting Eq. (9) to the B SBP are shown by a solid, dashed and dotted curve, respectively. c-d) logarithmic and $R^{* 1 / 4}$ representation of the SBP in $B$ and $R$, e) $B-R$ profile as obtained with method I and II (Sect. 6). f) position angle $\phi$ vs. $R^{*}$

We find that $\eta>1$ gives good fits. Values of $\eta<1$ give concave surface brightness distributions which cannot fit the plateau component ${ }^{1}$.

iii) The third component characterizes the inner part of SBPs of BCDs, which in general shows a luminosity excess over the plateau. It can be fitted by a gaussian distribution of the form:

$$
I_{\mathrm{G}}=I_{\mathrm{G}, 0} \exp \left[-0.5\left(R^{*} / \gamma\right)^{2}\right]
$$

which translates to

$$
\mu_{\mathrm{G}}=\mu_{\mathrm{G}}+0.54\left[\left(R^{*} / \gamma\right)^{2}\right]
$$

Because this component is usually very compact (size $<$ $10^{\prime \prime}$ ) the derived parameters (the central intensity $I_{\mathrm{G}, 0}$ and the scale length $\gamma$ ) can be seeing-dependent. Thus,

\footnotetext{
${ }^{1}$ We note that the functional form given by Eq. (5) is well suited for describing a wide variety of SBPs, ranging from a de Vaucouleurs law $(\eta=0.25)$ to an exponential law $(\eta=1)$. It has been found to provide a reasonably good approximation to SBPs of various types of galaxies (Cellone et al. 1994; James 1994; Young \& Currie 1994). For dEs, the exponent $\eta$ has been proposed to be used as a distance indicator (Young \& Currie 1994, 1995).
}

we shall avoid using them in our analysis of the structural parameters of BCDs (Paper II, Sect. 2) and consider instead only the integrated luminosity of this component.

To summarize, the surface brightness at a given radius can be written as:

$$
\mu\left(R^{*}\right)=-2.5 \cdot \log \left(I_{\mathrm{G}}+I_{\mathrm{P}}+I_{\mathrm{E}}\right)+\text { constant }
$$

The morphological transition from $\mathrm{iE}$ to $\mathrm{nE}$ type BCDs translates into a gradual decrease in the importance of the plateau component. Thus, only in the case of extreme $\mathrm{nE}$ BCDs, does a simplified 2-component model (Eqs. (3) and (5) with $\eta \leq 2$ ), produce fits of comparable quality to the 3-component model.

We shall characterize the radial extent of the HSB and of the LSB components respectively, by the radii $P_{25}$ of the plateau and $E_{25}$ of the exponential component, defined at a surface brightness level of $25 \mathrm{mag} \operatorname{arcsec}^{-2}$. We illustrate these radii on the SBP of Mkn 86 (Fig. 2b).

As pointed out in Sect. 2.3.1, from the morphological point of view, there are many intermediate types between the extreme examples of $\mathrm{iE} \mathrm{BCDs} \mathrm{(Mkn} \mathrm{86)} \mathrm{and} \mathrm{nE} \mathrm{BCDs}$ (Haro 2). In order to describe quantitatively this morphological variation we introduce the distance-independent 

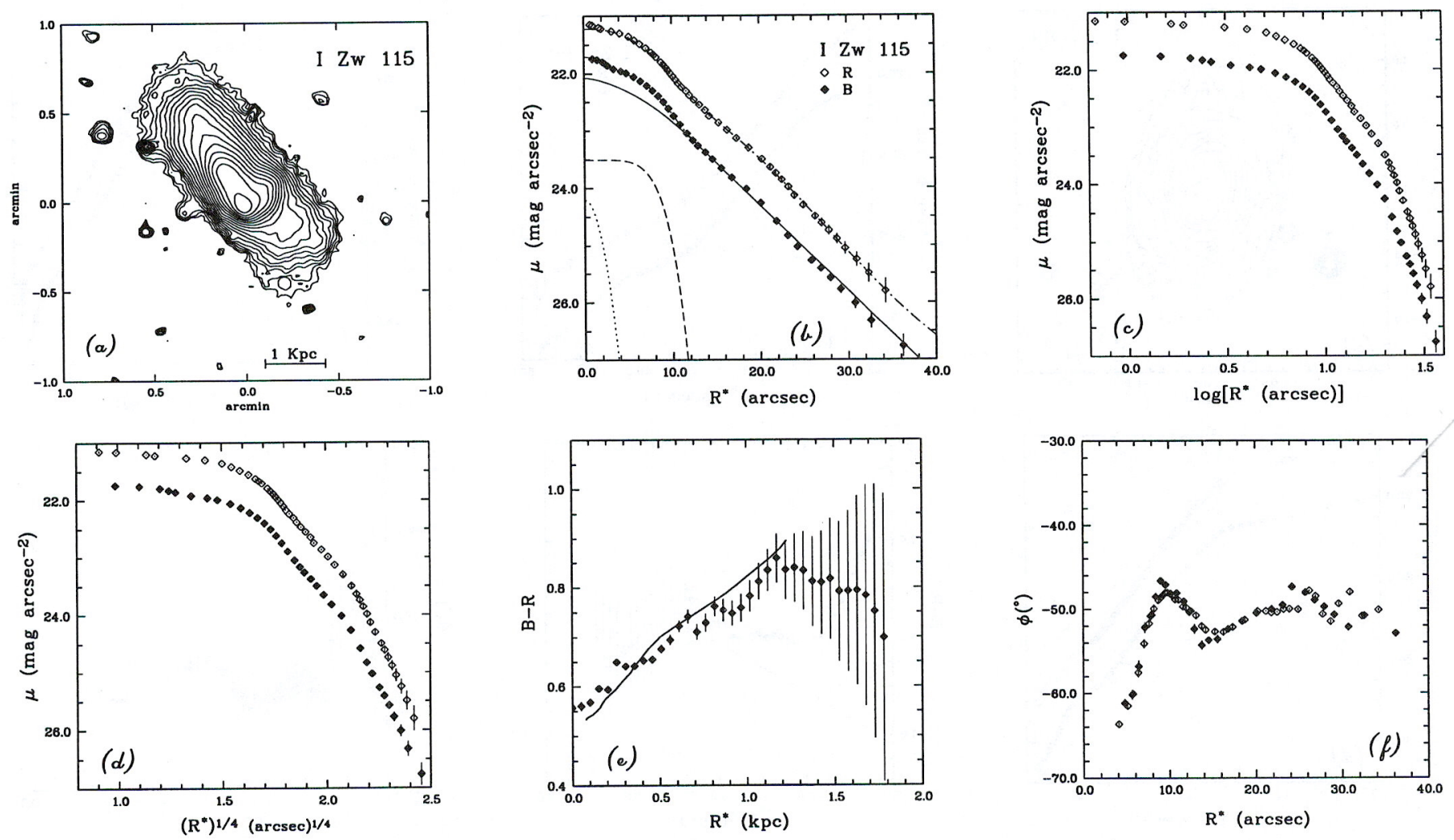

Fig. 6. I Zw 115: a) contour plot in $B$, from 22.0 to $26.0 \mathrm{mag} \operatorname{arcsec}^{-2}$ in steps of $0.25 \mathrm{mag}$, b) linear representation of the SBP in $B$ and $R$. The exponential, plateau and gaussian luminosity components as obtained by fitting Eq. (9) to the SBP are shown by a solid, dashed and dotted curve respectively. Note that instead of Eq. (3), we used Eq. $(22)(b, q=1.2,0.4)$ to describe the underlying LSB component. c-d) logarithmic and $R^{* 1 / 4}$ representation of the SBP in $B$ and $R$. e) $B-R$ profile as obtained with method I and II (Sect. 6). f) position angle $\phi$ vs. $R^{*}$

concentration index $C I$, a measure of the ratio of the area of the star-forming region to the total projected area of a $\mathrm{BCD}$ in the $B$ band.

$$
C I=1-\left(\frac{P_{25}}{E_{25}}\right)^{2}
$$

The $C I$ is given in Col. 6 of Table $4^{2}$. Along the morphological sequence of BCDs (Loose \& Thuan 1985) CI varies from $\gtrsim 0.8$ for BCDs with nuclear starburst (type $\mathrm{nE}$ ) to

\footnotetext{
${ }^{2}$ We prefer to use $C I$ rather than the King concentration parameter (King 1966) $c \equiv \log \left(R_{\mathrm{t}} / R_{\mathrm{c}}\right.$ ), where $R_{\mathrm{c}}$ is the core radius and $R_{\mathrm{t}}$ is the tidal radius, because $P_{25}$ and $R_{25}$ are much less sensitive to seeing effects than $R_{\mathrm{c}}$ and it can be better interpreted in the context of the decomposition scheme used here.

James (1991) has proposed another concentration parameter defined as $\log \left(R_{80} / R_{20}\right)$ where $R_{80}$ and $R_{20}$ denote the radii containing $80 \%$ and $20 \%$ of the total light of the galaxy. This concentration parameter or the one proposed by Okamura et al. (1980) (see also Doi et al. 1995) are more suited for nearinfrared profiles of BCDs which describe mainly the underlying component, than for the optical profiles, where the starburst component is more prominent.
}

$<0.5$ for those where the starburst activity is spread almost over the entire surface of the galaxy. The extreme BCD type i0 of Loose \& Thuan would have $C I \simeq 0$.

The apparent luminosity of an arbitrary component within its isophotal radius $R_{25}$ defined at $25 \mathrm{mag} \operatorname{arcsec}^{-2}$ can be obtained by

$$
m\left(R_{25}\right)=-2.5 \log \left(\int_{0}^{R_{25}} 2 \pi I\left(R^{*}\right) R^{*} \mathrm{~d} R^{*}\right)+\text { constant }
$$

The total apparent luminosity of the components described by Eqs. (3) and (5) is

$$
m\left(R_{\infty}\right)=-2.5 \log \left(2 \pi\left(\frac{\Gamma(2 / \eta)}{\eta}\right) I_{0} l^{2}\right)+\text { constant }
$$

where $I_{0}, l$ and $\eta$ are respectively the central intensity, scale length and exponent $(\eta=1$ for the exponential component and $\eta \geq 1$ for the plateau component) and $\Gamma$ is the Gamma-function. We also applied Eq. (9) to the $B$ and $R$ SBPs of additional BCDs from the literature: Haro 2 (Loose \& Thuan 1986) and NGC 2915 (Meurer et al. 1994). Throughout we utilized the iterative nonlinear fitting algorithm described by Marquardt (1963). 
This standard $\chi^{2}$-minimizing routine has proven to be efficient. With regard to the structural parameters $\mu_{\mathrm{E}, 0}$ and $\alpha$ of the LSB component it gave results in good agreement with those one would obtain from a linear extrapolation of the outer slope of the SBP inwards. Whenever error estimates were available, fits were performed twice, one weighting each point by its error bar (designated by W), and another with all points having equal weight (designated by $N$ ). We rejected fits with reduced $\chi^{2}>2$ and those giving an exponent $\eta<1$ in Eq. (5). Furthermore, we include in our analysis the data of Diehl (1988) for Mkn 36 and and the $B$ data for $M k n 5, M k n 7, M k n 49$, Mkn 400 and Mkn 487. As there were guiding problems with the exposure of $M k n 7$, we shall consider only the structural properties of its LSB component which are not affected by these difficulties.

\section{Results of the decomposition scheme}

We display the profile decomposition for the SBPs in the $B$-band for each of the above galaxies in Figs. $1 \mathrm{~b}-6 \mathrm{~b}$ for our sample and in Fig. 7 and Fig. 8 for the BCDs from other authors. The underlying, plateau and gaussian components are shown as solid, dashed and dotted lines, respectively.

The derived fit parameters along with their uncertainties as they were deduced from the fit are listed in Tables 2 and 3 . Secondary parameters, such as the radii $E_{25}$ and $P_{25}$ are given in Tables 4 and 5, and Tables 6 and 7 contain the absolute luminosities of the individual components within $R_{25}$. Errors included in Tables 4-7 denote the mean uncertainties of secondary properties as estimated from the uncertainties of the primary fit parameters. Relations between various quantities listed in Tables 2-7 and their physical implications will be discussed in Paper II.

\section{Deprojection of the three photometric components of the surface brightness profiles}

Understanding the starburst phenomenon in isolated dwarf galaxies requires the knowledge of the global gravitational potential within which the starburst activity occurs. In the absence of dark matter, the total mass of a $\mathrm{BCD}$ is the sum of its gas and stellar mass.

In this section we derive the radial luminosity density distribution of the stellar mass component. From the observable intensity distribution $I\left(R^{*}\right) \propto \operatorname{dex}\left(-0.4 \mu\left(R^{*}\right)\right)$ at the photometric radius $R^{*}$ the luminosity density $l(r)$ as a function of the intrinsic radius $r$ is obtained by deprojection. For a known mass-to-light ratio $\mathcal{M}(r) / \mathcal{L}(r)$, the stellar density distribution is then

$$
\rho(r)=\mathcal{M}(r) / \mathcal{L}(r) \cdot l(r)
$$

Although $l(r)$ can in principle be deduced directly by deprojection of a surface brightness profile, we did not do so, mainly because in that case the result cannot be interpreted in an unambiguous and systematic manner. The separate derivation of $l(r)$ for each of the luminosity components described in Sect. 3.1 can be justified in so far as these components can be considered to represent distinct entities with respect to extent and mean stellar type. This approach allows us to introduce dimensionless quantities and describe $l(r)$ in a simple way. Figure 9 illustrates the parameters to be derived for a spherically symmetric distribution where $r \leq r_{1}$ is the intrinsic radial variable. Assuming isotropy of the radiation, the emission coefficient $\epsilon(r)$ in units of erg s $\mathrm{cm}^{-1} \mathrm{~cm}^{-3} \mathrm{sr}^{-1}$ is $\epsilon(r)=l(r) / 4 \pi$ where $l(r)$ is in units of $\mathrm{erg} \mathrm{s}^{-1} \mathrm{~cm}^{-3}$. The line-of-sight variable $x$ ranging between $-x_{1}$ and $+x_{1}$ is related to the intrinsic radius $r \leq r_{1}$ and the photometric radius $R^{*}$ by $x= \pm\left(r^{2}-R^{* 2}\right)^{1 / 2}$.

In the absence of absorption or scattering, the equation of radiation transfer reads:

$$
\frac{\mathrm{d} I\left(x, R^{*}\right)}{\mathrm{d} x}=\epsilon(r)=\frac{l(r)}{4 \pi}
$$

Since $\mathrm{d} I / \mathrm{d} x=0$ for $-x_{1} \geq x$ and $x>+x_{1}$, Eq. (14) can be written in terms of the Abel integral equation

$$
I\left(R^{*}\right)=\frac{1}{2 \pi} \int_{R^{*}}^{r_{1}} \frac{r l(r)}{\left(r^{2}-R^{* 2}\right)^{1 / 2}} \mathrm{~d} r
$$

From the inversion of Eq. (15) the intrinsic luminosity density obtains as follows:

$$
\begin{aligned}
l(r) & =-\frac{4}{r} \frac{\mathrm{d}}{\mathrm{dr}} \zeta(r) & & (r>0) \\
l(r) & =-8 \frac{\mathrm{d}^{2}}{\mathrm{dr}^{2}} \zeta(r) & & (r=0)
\end{aligned}
$$

where $\zeta(r)$ is given by:

$$
\zeta(r)=\int_{r}^{r_{1}} \frac{R^{*} I\left(R^{*}\right)}{\left(R^{* 2}-r^{2}\right)^{1 / 2}} \mathrm{~d} R^{*}
$$

Utilizing the scale length $\alpha$ of an intensity distribution $I\left(R^{*}\right)$, it is convenient to introduce the dimensionless radius $y=r / \alpha$ and luminosity density $L(y)$

$$
L(y)=-\frac{4}{y} \frac{\mathrm{d}}{\mathrm{dy}} \zeta(y)
$$

The intrinsic luminosity density is then $l(y)=\left(I_{0} / \alpha\right) L(y)$ where $I_{0}$ is the intensity at $y=0$.

Except for some simple cases (e.g. a power-law intensity distribution) the integral in Eq. (18) has to be computed numerically. Usually the integration has been performed out to $y \gtrsim 10$ which encloses typically more than $99 \%$ of the flux of the intensity distribution. We used throughout a $4^{\text {th }}$ order Runge-Kutta method with estimation and correction of the local error and a variable step size. The derivative $\mathrm{d} \zeta(r) / \mathrm{d} r$ has been calculated 
Table 2. Derived parameters in the $B$ band $^{a}$

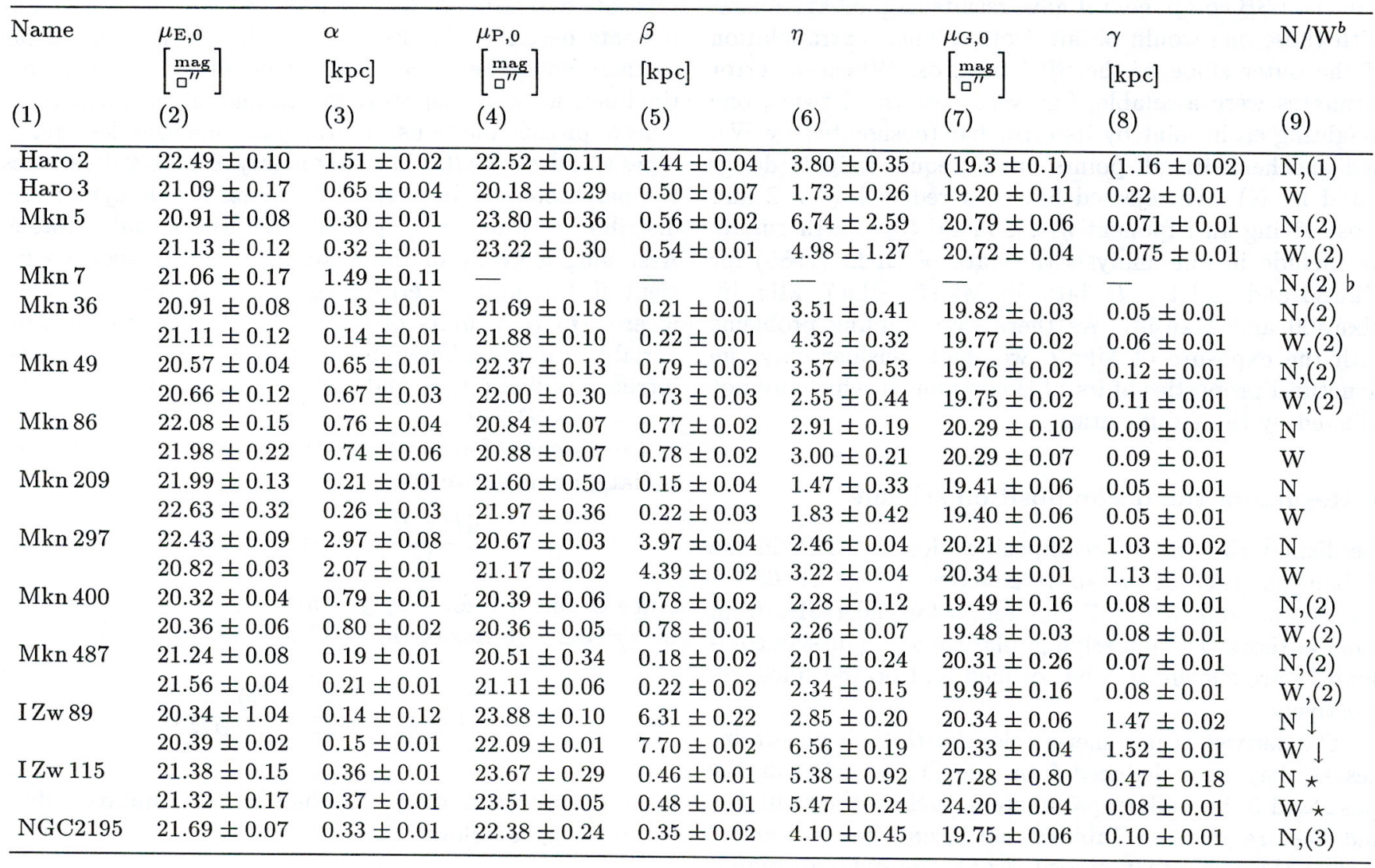

$a$ : Fit parameters derived by applying the 3-component model Eq. (9) to the SBPs of BCDs in the $B$ band, corrected for galactic foreground absorption.

$b: \mathrm{N} \equiv$ non-weighted fit; $\mathrm{W} \equiv$ weighted fit

(1): SBP from Loose \& Thuan (1986). Photometry has been published for radii $\gtrsim 6^{\prime \prime}$, thus only indicative parameters could be deduced for the gaussian component (Cols. 7 and 8).

(2): Surface photometry and decomposition according to Eq. (9) from Diehl (1988).

(3): SBP by Meurer et al. (1994). From the velocity of NGC 2915 relative to the Local Group (Reif et al. 1982) and with a Hubble constant of $75 \mathrm{~km} \mathrm{~s}^{-1} \mathrm{Mpc}^{-1}$ we adopt $D_{\mathrm{NGC} 2915}=2.7 \mathrm{Mpc}$.

b: Due to guiding problems, the SBP flattens near the center, making the parameters of the gaussian and plateau components uncertain. Therefore only the parameters of the LSB component are listed.

*: The underlying BCD component has been modeled by Eq. $(22)$ with $(b, q)=(1.2,0.4)$ instead of a pure exponential law (Eq. 3 ). l: The SBP of the non-BCD spiral galaxy IZw 89 can be formally described by Eq. (9). However the resulting parameters are very dissimilar to those derived for BCDs.

from cubic spline interpolation. To check our results, we carried out the procedure in the reverse direction, i.e. we re-projected $l(y)$ and compared the results with the initial intensity distribution. We always found good agreement. In the following sections we discuss the intrinsic luminosity densities for the 3 components considered in the decomposition scheme.

\subsection{Deprojection of the gaussian component}

A gaussian intensity distribution is projection-invariant. The luminosity density and projected intensity are related by the following equations:

$$
\begin{gathered}
I\left(R^{*}\right)=I_{0} \exp \left(-\frac{R^{* 2}}{\gamma^{2}}\right)=\frac{\gamma l_{0} \Gamma(1.5)}{2 \pi} \exp \left(-\frac{R^{* 2}}{\gamma^{2}}\right) \\
l(r)=l_{0} \exp \left(-\frac{r^{2}}{\gamma^{2}}\right)=\frac{2 \pi I_{0}}{\gamma \Gamma(1.5)} \exp \left(-\frac{r^{2}}{\gamma^{2}}\right)
\end{gathered}
$$


Table 3. Derived parameters in the $R$ band

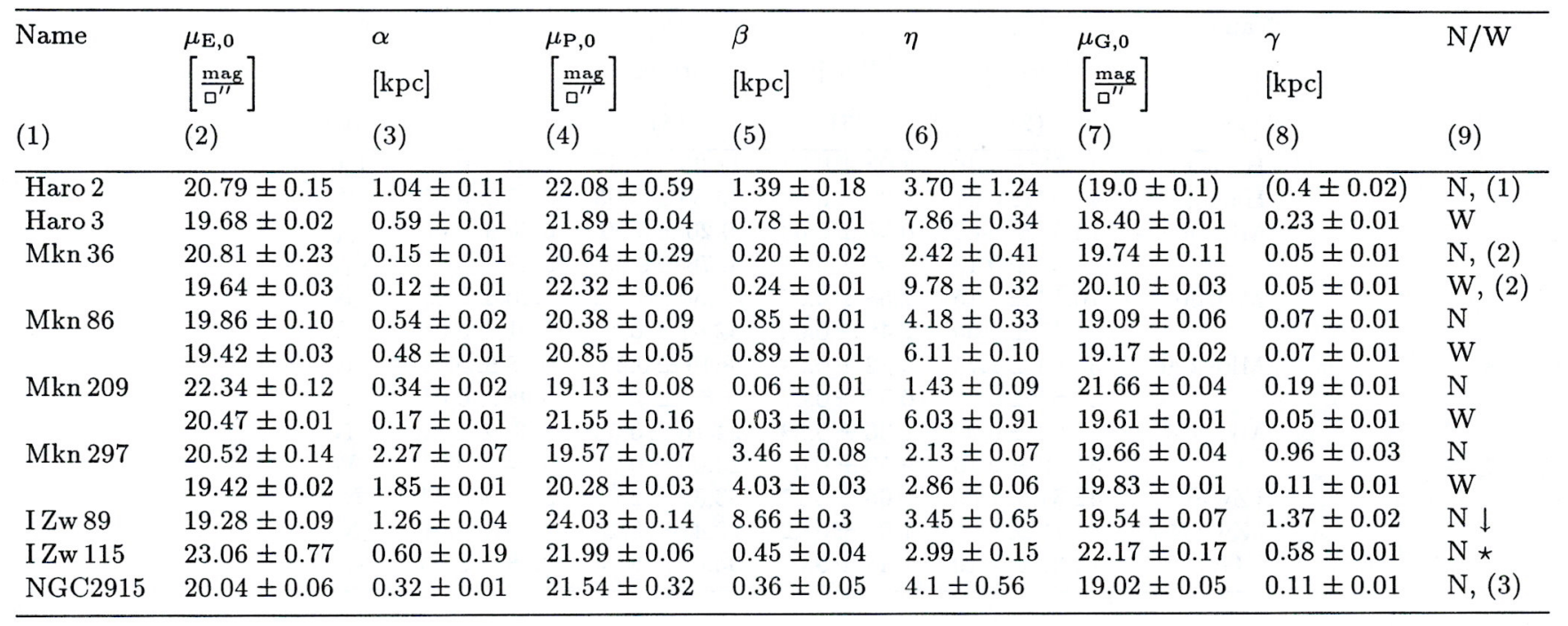

See notes in Table 2 .

Table 4. Extent of the starburst and underlying components in the $B$ band

\begin{tabular}{|c|c|c|c|c|c|c|}
\hline Name & $\begin{array}{c}E_{25} \\
{[\operatorname{arcsec}]}\end{array}$ & $\begin{array}{c}E_{25} \\
{[\mathrm{kpc}]}\end{array}$ & $\begin{array}{c}P_{25} \\
{[\operatorname{arcsec}]}\end{array}$ & $\begin{array}{c}P_{25} \\
{[\mathrm{kpc}]}\end{array}$ & $C I$ & $\mathrm{~W} / \mathrm{N}$ \\
\hline (1) & $(2)$ & (3) & (4) & (5) & (6) & (7) \\
\hline Haro 2 & $35.16 \pm 1.14$ & $3.49 \pm 0.11$ & $17.99 \pm 0.40$ & $1.79 \pm 0.04$ & $0.74 \pm 0.08$ & $\mathrm{~N},(1)$ \\
\hline Haro 3 & $34.88 \pm 1.79$ & $2.35 \pm 0.12$ & $17.72 \pm 2.39$ & $1.19 \pm 0.16$ & $0.74 \pm 0.06$ & W' \\
\hline \multirow[t]{2}{*}{ Mkn 5} & $17.63 \pm 0.39$ & $1.14 \pm 0.02$ & $8.55 \pm 0.37$ & $0.55 \pm 0.02$ & $0.76 \pm 0.02$ & $\mathrm{~N},(2)$ \\
\hline & $17.93 \pm 0.64$ & $1.16 \pm 0.04$ & $9.01 \pm 0.32$ & $0.59 \pm 0.02$ & $0.75 \pm 0.03$ & $\mathrm{~W},(2)$ \\
\hline Mkn 7 & $25.89 \pm 1.48$ & $5.39 \pm 0.31$ & - & - & - & $\mathrm{N}$ \\
\hline \multirow[t]{2}{*}{ Mkn 36} & $12.35 \pm 0.23$ & $0.48 \pm 0.01$ & $7.38 \pm 0.31$ & $0.29 \pm 0.01$ & $0.64 \pm 0.03$ & $\mathrm{~N},(2)$ \\
\hline & $12.55 \pm 0.44$ & $0.49 \pm 0.02$ & $7.39 \pm 0.13$ & $0.29 \pm 0.01$ & $0.65 \pm 0.03$ & $\mathrm{~W},(2)$ \\
\hline \multirow[t]{2}{*}{ Mkn 49} & $29.96 \pm 0.30$ & $2.64 \pm 0.03$ & $11.48 \pm 0.41$ & $1.01 \pm 0.04$ & $0.85 \pm 0.01$ & $\mathrm{~N},(2)$ \\
\hline & $30.20 \pm 1.12$ & $2.66 \pm 0.10$ & $12.34 \pm 0.78$ & $1.09 \pm 0.07$ & $0.83 \pm 0.02$ & $\mathrm{~W},(2)$ \\
\hline \multirow[t]{2}{*}{ Mkn 86} & $60.53 \pm 3.27$ & $2.06 \pm 0.11$ & $36.20 \pm 0.97$ & $1.23 \pm 0.03$ & $0.64 \pm 0.04$ & $\mathrm{~N}$ \\
\hline & $60.36 \pm 4.78$ & $2.05 \pm 0.16$ & $35.82 \pm 0.94$ & $1.22 \pm 0.03$ & $0.65 \pm 0.06$ & W \\
\hline \multirow[t]{2}{*}{ Mkn 209} & $25.73 \pm 1.03$ & $0.57 \pm 0.02$ & $15.08 \pm 2.38$ & $0.34 \pm 0.05$ & $0.66 \pm 0.11$ & $\mathrm{~N}$ \\
\hline & $25.05 \pm 3.27$ & $0.56 \pm 0.07$ & $17.38 \pm 2.69$ & $0.39 \pm 0.06$ & $0.52 \pm 0.08$ & W \\
\hline \multirow[t]{2}{*}{ Mkn 297} & $23.04 \pm 0.74$ & $7.04 \pm 0.23$ & $22.82 \pm 0.23$ & $6.97 \pm 0.07$ & $0.02 \pm 0.06$ & $\mathrm{~N}$ \\
\hline & $26.16 \pm 0.19$ & $7.99 \pm 0.06$ & $21.24 \pm 0.10$ & $6.49 \pm 0.03$ & $0.34 \pm 0.01$ & W \\
\hline \multirow[t]{2}{*}{ Mkn 400} & $21.67 \pm 0.21$ & $3.39 \pm 0.03$ & $9.42 \pm 0.26$ & $1.48 \pm 0.04$ & $0.81 \pm 0.01$ & $\mathrm{~N},(2)$ \\
\hline & $21.78 \pm 0.02$ & $3.41 \pm 0.06$ & $9.50 \pm 0.15$ & $1.49 \pm 0.02$ & $0.81 \pm 0.01$ & $\mathrm{~W},(2)$ \\
\hline \multirow[t]{2}{*}{ Mkn 487} & $12.63 \pm 0.26$ & $0.67 \pm 0.01$ & $6.90 \pm 0.70$ & $0.36 \pm 0.04$ & $0.70 \pm 0.06$ & $\mathrm{~N},(2)$ \\
\hline & $12.81 \pm 0.40$ & $0.68 \pm 0.02$ & $7.25 \pm 0.45$ & $0.38 \pm 0.02$ & $0.68 \pm 0.04$ & $\mathrm{~W},(2)$ \\
\hline \multirow[t]{2}{*}{ I Zw 89} & $3.72 \pm 2.63$ & $0.58 \pm 0.41$ & $40.64 \pm 0.03$ & $6.38 \pm 0.20$ & $<0$ & $N \downarrow$ \\
\hline & $4.02 \pm 0.01$ & $0.63 \pm 0.01$ & $56.94 \pm 0.22$ & $8.94 \pm 0.03$ & $<0$ & $\mathrm{~W} \downarrow$ \\
\hline \multirow[t]{2}{*}{ I Zw 115} & $23.41 \pm 0.76$ & $1.19 \pm 0.04$ & $9.47 \pm 0.33$ & $0.48 \pm 0.02$ & $0.83 \pm 0.01$ & $N \star$ \\
\hline & $24.68 \pm 0.90$ & $1.26 \pm 0.05$ & $9.98 \pm 0.06$ & $0.51 \pm 0.00$ & $0.84 \pm 0.02$ & $\mathrm{~W} \star$ \\
\hline NGC 2915 & $75.61 \pm 1.48$ & $1.00 \pm 0.02$ & $33.20 \pm 1.44$ & $0.44 \pm 0.02$ & $0.81 \pm 0.02$ & $\mathrm{~N},(3)$ \\
\hline
\end{tabular}

See notes in Table 2. 
Table 5. Extent of the starburst and underlying components in the $R$ band

\begin{tabular}{lccccc}
\hline Name & $\begin{array}{c}E_{25} \\
{[\operatorname{arcsec}]}\end{array}$ & $\begin{array}{c}E_{25} \\
{[\mathrm{kpc}]}\end{array}$ & $\begin{array}{c}P_{25} \\
{[\operatorname{arcsec}]}\end{array}$ & $\begin{array}{c}P_{25} \\
{[\mathrm{kpc}]}\end{array}$ & $\mathrm{W} / \mathrm{N}$ \\
& $(2)$ & $(3)$ & $(4)$ & $(5)$ & $(6)$ \\
\hline 1) & $40.55 \pm 3.46$ & $4.03 \pm 0.34$ & $18.29 \pm 2.34$ & $1.82 \pm 0.23$ & $\mathrm{~N},(1)$ \\
Haro 2 & $43.03 \pm 0.31$ & $2.90 \pm 0.02$ & $13.30 \pm 0.06$ & $0.90 \pm 0.01$ & $\mathrm{~W}$ \\
Mkaro 3 & $14.96 \pm 0.88$ & $0.58 \pm 0.03$ & $9.20 \pm 0.90$ & $0.36 \pm 0.03$ & $\mathrm{~N},(2)$ \\
& $14.62 \pm 0.13$ & $0.57 \pm 0.01$ & $6.78 \pm 0.05$ & $0.27 \pm 0.01$ & $\mathrm{~W},(2)$ \\
Mkn 86 & $76.10 \pm 2.15$ & $2.58 \pm 0.07$ & $35.30 \pm 0.82$ & $1.20 \pm 0.03$ & $\mathrm{~N}$ \\
& $72.46 \pm 0.65$ & $2.46 \pm 0.02$ & $32.67 \pm 0.15$ & $1.11 \pm 0.01$ & $\mathrm{~W}$ \\
Mkn 209 & $36.86 \pm 1.75$ & $0.82 \pm 0.04$ & $8.16 \pm 0.53$ & $0.18 \pm 0.01$ & $\mathrm{~N}$ \\
& $31.62 \pm 0.85$ & $0.70 \pm 0.02$ & $1.69 \pm 0.04$ & $0.04 \pm 0.01$ & $\mathrm{~W}$ \\
Mkn 297 & $30.63 \pm 0.97$ & $9.36 \pm 0.29$ & $24.16 \pm 0.56$ & $7.38 \pm 0.17$ & $\mathrm{~N}$ \\
& $31.09 \pm 0.16$ & $9.50 \pm 0.05$ & $22.06 \pm 0.21$ & $6.74 \pm 0.06$ & $\mathrm{~W}$ \\
IZw 89 & $42.32 \pm 1.08$ & $6.65 \pm 0.17$ & $53.32 \pm 2.02$ & $8.37 \pm 0.32$ & $\mathrm{~N} \downarrow$ \\
IZw 115 & $21.13 \pm 8.25$ & $1.08 \pm 0.42$ & $12.47 \pm 0.18$ & $0.63 \pm 0.01$ & $\mathrm{~N} \star$ \\
NGC 2915 & $112.6 \pm 2.10$ & $1.48 \pm 0.03$ & $36.08 \pm 2.06$ & $0.48 \pm 0.03$ & $\mathrm{~N},(3)$ \\
\hline
\end{tabular}

See notes in Table 2.

Table 6. Absolute luminosities of the components within $R_{25}$ in $B$

\begin{tabular}{|c|c|c|c|c|c|c|c|}
\hline Name & $\begin{array}{l}M_{\mathrm{E}} \\
{[\mathrm{mag}]}\end{array}$ & $\begin{array}{l}M_{\mathrm{P}} \\
{[\mathrm{mag}]}\end{array}$ & $\begin{array}{l}M_{\mathrm{G}} \\
\text { [mag] }\end{array}$ & $\begin{array}{l}M_{\mathrm{G}+\mathrm{P}} \\
{[\mathrm{mag}]}\end{array}$ & $\begin{array}{l}M_{\mathrm{fit}} \\
{[\mathrm{mag}]}\end{array}$ & $\frac{L_{\mathrm{G}+\mathrm{P}}}{L_{\mathrm{E}}}$ & $\mathrm{W} / \mathrm{N}$ \\
\hline (1) & $(2)$ & (3) & (4) & (5) & (6) & (7) & (8) \\
\hline Haro 2 & $-16.59 \pm 0.11$ & $-15.92 \pm 0.09$ & $-17.04 \pm 0.06$ & $-17.37 \pm 0.05$ & $-17.80 \pm 0.04$ & 3.05 & $\mathrm{~N}(1)$ \\
\hline Haro 3 & $-16.40 \pm 0.17$ & $-16.21 \pm 0.29$ & $-16.05 \pm 0.12$ & $-16.89 \pm 0.16$ & $-17.50 \pm 0.10$ & 1.57 & $\mathrm{~W}$ \\
\hline Mkn 5 & $\begin{array}{l}9 \pm 0.08 \\
0 \pm 0.12\end{array}$ & $\begin{array}{l}-12.63 \pm 0.29 \\
-13.12 \pm 0.23\end{array}$ & $\begin{array}{l}-12.03 \pm 0.12 \\
-12.22 \pm 0.04\end{array}$ & $\begin{array}{l}-13.12 \pm 0.18 \\
-13.51 \pm 0.16\end{array}$ & $\begin{array}{l}-15.09 \pm 0.07 \\
-15.09 \pm 0.09\end{array}$ & $\begin{array}{l}0.19 \\
0.30\end{array}$ & $\begin{array}{l}\mathrm{N},(2) \\
\mathrm{W},(2)\end{array}$ \\
\hline Mkn 7 & $\begin{array}{l}-14.80 \pm 0.12 \\
-18.22 \pm 0.19\end{array}$ & $\begin{array}{l}-13.12 \pm 0.23 \\
-\end{array}$ & - & $-18.21 \pm 0.18$ & $-18.97 \pm 0.05$ & 0.99 & $\mathrm{Nb}$ \\
\hline Mkn 36 & $-13.05 \pm 0.07$ & $-12.60 \pm 0.14$ & $-12.35 \pm 0.10$ & $-13.23 \pm 0.08$ & $-13.90 \pm 0.05$ & 1.18 & $\mathrm{~N},(2)$ \\
\hline & $-12.97 \pm 0.12$ & $-12.56 \pm 0.07$ & $-12.52 \pm 0.05$ & $-13.29 \pm 0.07$ & $-13.90 \pm 0.05$ & 1.36 & $\mathrm{~W},(2)$ \\
\hline Mkn 49 & $\begin{array}{l}-16.96 \pm 0.04 \\
-16.92 \pm 0.12\end{array}$ & $\begin{array}{l}-14.81 \pm 0.10 \\
-15.05 \pm 0.23\end{array}$ & $\begin{array}{l}-14.13 \pm 0.05 \\
-14.09 \pm 0.03\end{array}$ & $\begin{array}{l}-15.28 \pm 0.06 \\
-15.43 \pm 0.16\end{array}$ & $\begin{array}{l}-17.16 \pm 0.03 \\
-17.17 \pm 0.10\end{array}$ & $\begin{array}{l}0.21 \\
0.25\end{array}$ & $\begin{array}{l}\mathrm{N},(2) \\
\mathrm{W},(2)\end{array}$ \\
\hline Mkn 86 & $-15.55 \pm 0.17$ & $-16.31 \pm 0.06$ & $-13.14 \pm 0.24$ & $-16.37 \pm 0.06$ & $-16.79 \pm 0.05$ & 2.13 & $\mathrm{~N}$ \\
\hline Mkn 209 & $-15.60 \pm 0.25$ & $-16.29 \pm 0.06$ & $-13.15 \pm 0.17$ & $-16.34 \pm 0.06$ & $-16.79 \pm 0.08$ & 1.98 & W \\
\hline & $\begin{array}{l}-12.86 \pm 0.13 \\
-12.50 \pm 0.39\end{array}$ & $\begin{array}{l}-12.27 \pm 0.41 \\
-12.53 \pm 0.35\end{array}$ & $\begin{array}{l}-12.41 \pm 0.09 \\
-12.57 \pm 0.12\end{array}$ & $\begin{array}{l}-13.10 \pm 0.19 \\
-13.30 \pm 0.17\end{array}$ & $\begin{array}{l}-13.74 \pm 0.11 \\
-13.73 \pm 0.14\end{array}$ & $\begin{array}{l}1.24 \\
2.09\end{array}$ & $\begin{array}{l}\mathrm{N} \\
\mathrm{W}\end{array}$ \\
\hline Mkn 297 & $-18.09 \pm 0.10$ & $-20.08 \pm 0.03$ & $-18.41 \pm 0.05$ & $-20.29 \pm 0.02$ & $-20.42 \pm 0.02$ & 7.58 & $\mathrm{~N}$ \\
\hline M & $-19.22 \pm 0.03$ & $-19.72 \pm 0.02$ & $-18.48 \pm 0.01$ & $-20.02 \pm 0.01$ & $-20.44 \pm 0.01$ & 2.09 & $\mathrm{~W}$ \\
\hline Mkn 400 & $\begin{array}{l}-17.64 \pm 0.04 \\
-17.63 \pm 0.06\end{array}$ & $-16.84 \pm 0.05$ & $-13.50 \pm 0.23$ & $-16.89 \pm 0.05$ & $-18.08 \pm 0.03$ & 0.50 & $\mathrm{~N},(2)$ \\
\hline Mkn 487 & $\begin{array}{l}-17.63 \pm 0.06 \\
-13.59 \pm 0.08\end{array}$ & $-16.87 \pm 0.04$ & $-13.51 \pm 0.04$ & $-16.92 \pm 0.04$ & $-18.08 \pm 0.03$ & 0.52 & $\mathrm{~W},(2)$ \\
\hline & $\begin{array}{l}-13.59 \pm 0.08 \\
-13.45 \pm 0.07\end{array}$ & $\begin{array}{l}-13.58 \pm 0.29 \\
-13.38 \pm 0.12\end{array}$ & $\begin{array}{l}-12.55 \pm 0.27 \\
-13.17 \pm 0.22\end{array}$ & $\begin{array}{l}-13.94 \pm 0.21 \\
-14.03 \pm 0.10\end{array}$ & $\begin{array}{l}-14.53 \pm 0.12 \\
-14.53 \pm 0.07\end{array}$ & $\begin{array}{l}1.38 \\
1.71\end{array}$ & $\begin{array}{l}\mathrm{N},(2) \\
\mathrm{W},(2)\end{array}$ \\
\hline I Zw 89 & $-13.90 \pm 2.43$ & $-17.81 \pm 0.11$ & $-19.06 \pm 0.06$ & $-19.36 \pm 0.05$ & $-19.37 \pm 0.05$ & 152 & $\mathrm{~N} \downarrow$ \\
\hline & $-13.82 \pm 0.02$ & $-17.55 \pm 0.01$ & $-19.05 \pm 0.01$ & $-19.29 \pm 0.01$ & $-19.30 \pm 0.01$ & 2.09 & $\mathrm{~W} \downarrow$ \\
\hline NGC 2915 & $\begin{array}{l}-14.91 \pm 0.15 \\
-14.22 \pm 0.07\end{array}$ & $\begin{array}{l}-12.51 \pm 0.04 \\
-13.01 \pm 0.19\end{array}$ & $\begin{array}{l}-8.28 \pm 0.10 \\
-13.71 \pm 0.13\end{array}$ & & $\begin{array}{l}-15.03 \pm 0.13 \\
-14.94 \pm 0.05\end{array}$ & $\begin{array}{l}0.11 \\
0.95\end{array}$ & $\begin{array}{l}\mathrm{W} \star \\
\mathrm{N},(3)\end{array}$ \\
\hline
\end{tabular}

See notes in Table 2. 
Table 7. Absolute luminosities of the components within $R_{25}$ in $R$

\begin{tabular}{|c|c|c|c|c|c|c|c|}
\hline Name & $\begin{array}{l}M_{\mathrm{E}} \\
{[\mathrm{mag}]}\end{array}$ & $\begin{array}{l}M_{\mathrm{P}} \\
{[\mathrm{mag}]}\end{array}$ & $\begin{array}{l}M_{\mathrm{G}} \\
{[\mathrm{mag}]}\end{array}$ & $\begin{array}{l}M_{\mathrm{G}+\mathrm{P}} \\
{[\mathrm{mag}]}\end{array}$ & $\begin{array}{l}M_{\text {fit }} \\
{[\mathrm{mag}]}\end{array}$ & $\frac{L_{\mathrm{G}+\mathrm{P}}}{L_{\mathrm{E}}}$ & $\mathrm{W} / \mathrm{N}$ \\
\hline (1) & $(2)$ & $(3)$ & (4) & (5) & (6) & (7) & (8) \\
\hline Haro 2 & $-17.74 \pm 0.20$ & $-16.30 \pm 0.47$ & $-17.51 \pm 0.10$ & $-17.81 \pm 0.12$ & $-18.53 \pm 0.10$ & 2.07 & $\mathrm{~N},(1)$ \\
\hline Haro 3 & $-17.70 \pm 0.02$ & $-15.28 \pm 0.03$ & $-16.98 \pm 0.01$ & $-17.18 \pm 0.01$ & $-18.22 \pm 0.01$ & 0.62 & W \\
\hline Mkn 36 & $\begin{array}{l}-13.53 \pm 0.22 \\
-14.18 \pm 0.03\end{array}$ & $\begin{array}{l}-13.63 \pm 0.24 \\
-12.33 \pm 0.04\end{array}$ & $\begin{array}{l}-12.28 \pm 0.28 \\
-12.16 \pm 0.05\end{array}$ & $\begin{array}{l}-13.90 \pm 0.18 \\
-13.00 \pm 0.02\end{array}$ & $\begin{array}{l}-14.49 \pm 0.12 \\
-14.49 \pm 0.02\end{array}$ & $\begin{array}{l}1.41 \\
0.34\end{array}$ & $\begin{array}{l}\mathrm{N},(2) \\
\mathrm{W},(2)\end{array}$ \\
\hline Mkn 86 & $\begin{array}{l}-17.33 \pm 0.09 \\
-17.51 \pm 0.03\end{array}$ & $\begin{array}{l}-16.94 \pm 0.07 \\
-16.60 \pm 0.04\end{array}$ & $\begin{array}{l}-13.82 \pm 0.12 \\
-13.49 \pm 0.04\end{array}$ & $\begin{array}{l}-17.00 \pm 0.07 \\
-16.66 \pm 0.04\end{array}$ & $\begin{array}{l}-17.93 \pm 0.05 \\
-17.92 \pm 0.02\end{array}$ & $\begin{array}{l}0.74 \\
0.46\end{array}$ & $\begin{array}{l}\mathrm{N} \\
\mathrm{W}\end{array}$ \\
\hline Mkn 209 & $\begin{array}{l}-13.47 \pm 0.14 \\
-14.15 \pm 0.06\end{array}$ & $\begin{array}{l}-12.63 \pm 0.10 \\
-8.61 \pm 0.13\end{array}$ & $\begin{array}{l}-13.26 \pm 0.04 \\
-12.25 \pm 0.02\end{array}$ & $\begin{array}{l}-13.75 \pm 0.04 \\
-12.28 \pm 0.02\end{array}$ & $\begin{array}{l}-14.37 \pm 0.06 \\
-14.33 \pm 0.05\end{array}$ & $\begin{array}{l}1.29 \\
0.18\end{array}$ & $\begin{array}{l}\mathrm{N} \\
\mathrm{W}\end{array}$ \\
\hline Mkn 297 & $\begin{array}{l}-19.72 \pm 0.13 \\
-20.44 \pm 0.02\end{array}$ & $\begin{array}{l}-20.91 \pm 0.06 \\
-20.45 \pm 0.03\end{array}$ & $\begin{array}{l}-18.80 \pm 0.10 \\
-19.00 \pm 0.03\end{array}$ & $\begin{array}{l}-21.05 \pm 0.05 \\
-20.71 \pm 0.02\end{array}$ & $\begin{array}{l}-21.33 \pm 0.04 \\
-21.33 \pm 0.01\end{array}$ & $\begin{array}{l}3.42 \\
1.27\end{array}$ & $\begin{array}{l}\mathrm{N} \\
\mathrm{W}\end{array}$ \\
\hline I Zw 89 & $-19.79 \pm 0.09$ & $-18.44 \pm 0.15$ & $-19.71 \pm 0.07$ & $-20.00 \pm 0.05$ & $-20.65 \pm 0.05$ & 1.22 & $\mathrm{~N} \downarrow$ \\
\hline I Zw 115 & $\begin{array}{l}-13.71 \pm 1.12 \\
-14.85 \pm 0.12\end{array}$ & $\begin{array}{l}-14.02 \pm 0.04 \\
-13.35 \pm 0.06\end{array}$ & $\begin{array}{l}-15.13 \pm 0.16 \\
-14.78 \pm 0.19\end{array}$ & $\begin{array}{l}-15.47 \pm 0.12 \\
-15.03 \pm 0.15\end{array}$ & $\begin{array}{l}-15.66 \pm 0.20 \\
-15.70 \pm 0.08\end{array}$ & $\begin{array}{l}5.02 \\
1.18\end{array}$ & $\begin{array}{l}\mathrm{N} \star \\
\mathrm{W} \star\end{array}$ \\
\hline NGC 2915 & $-16.02 \pm 0.06$ & $-13.90 \pm 0.25$ & $-14.67 \pm 0.13$ & $-15.10 \pm 0.09$ & $-16.41 \pm 0.05$ & 0.43 & $\mathrm{~N},(3)$ \\
\hline
\end{tabular}

See notes in Table 2.
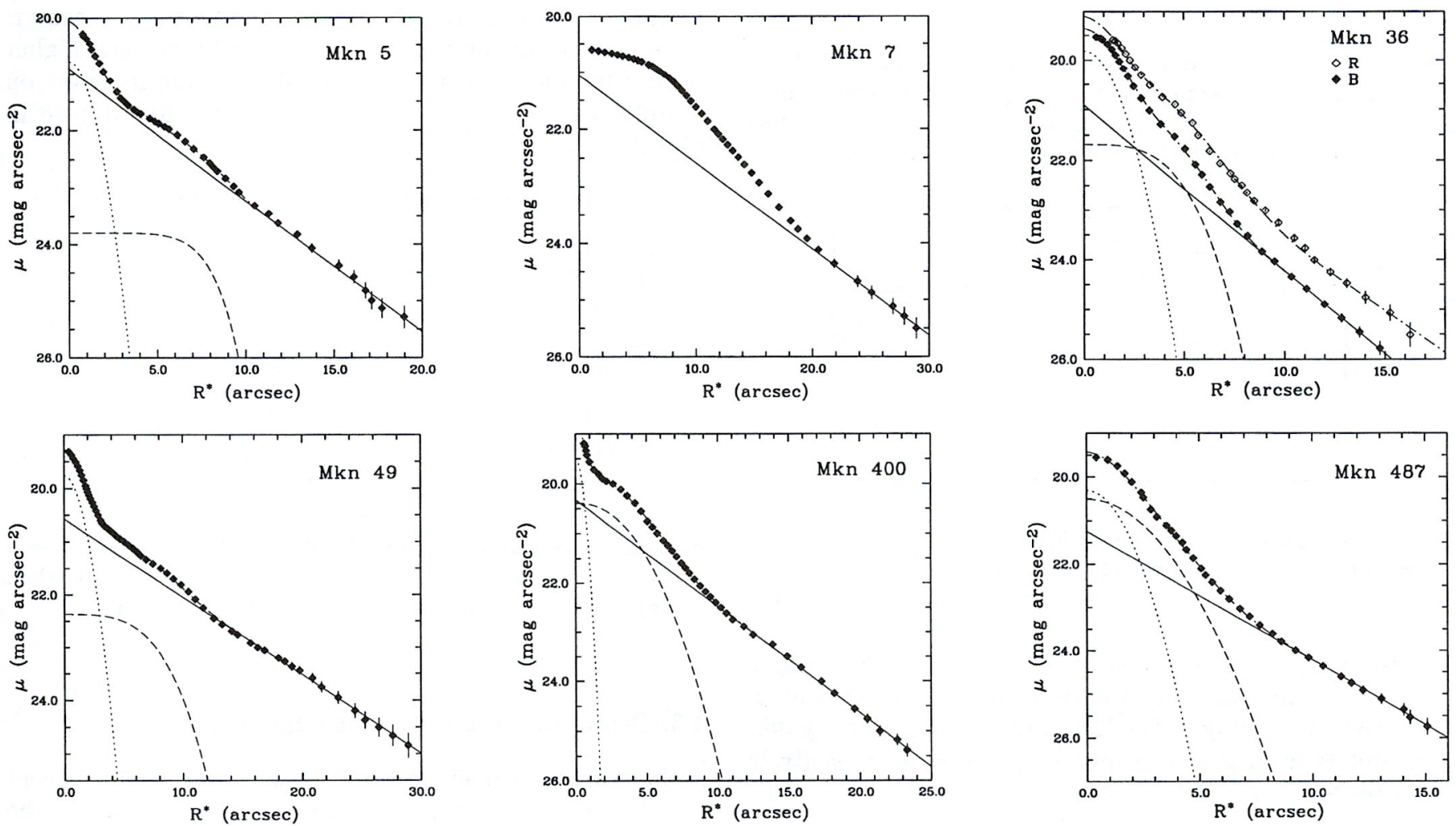

Fig. 7. Fits obtained by Diehl (1988) for the BCDs Mkn 5, Mkn 7, Mkn 36, Mkn 49, Mkn 400 and Mkn 487 according to the 3-component decomposition scheme (Eq. 9). The exponential, plateau and gaussian components are illustrated by a solid, dashed and dotted curve, respectively. For $\mathrm{Mkn} 7$, we consider only the exponential component which is not affected by the guiding problems described in the text 

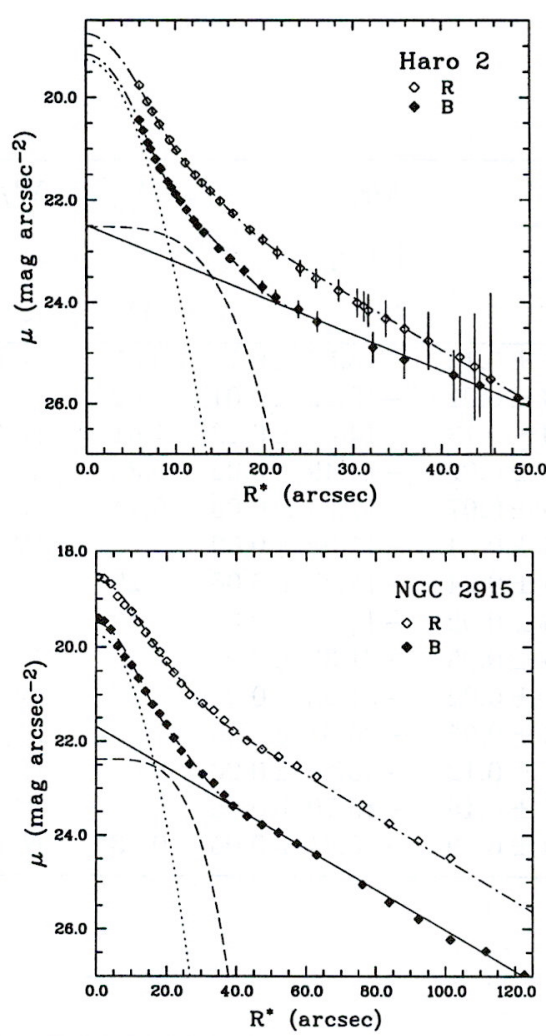

Fig. 8. Fits obtained for the BCDs Haro 2 and NGC 2915 according to the 3-component decomposition scheme (Eq.9). The exponential, plateau and gaussian components are illustrated by a solid, dashed and dotted curve, respectively

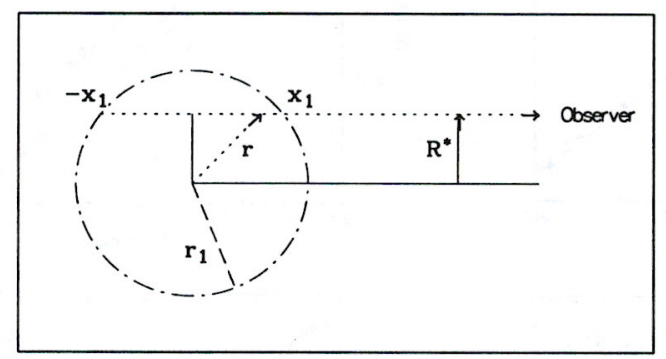

Fig. 9. Spherically symmetric luminosity distribution. The parameters $r$ and $R^{*}$ in Eq. (15) are indicated

Here $l_{0}$ and $I_{0}$ denote respectively the central luminosity density and intensity, and $\Gamma$ is the Gammafunction. According to Eq. (20) a gaussian luminosity density differs from the corresponding projected intensity by a factor $2 \pi \gamma^{-1} \Gamma(1.5)^{-1} \simeq 7.09 \gamma^{-1}$.

\subsection{Deprojection of the plateau component}

Figure 10 shows the dimensionless intensity $I(y) / I_{0}$ and luminosity density $L(y)=\beta \eta^{-1} I_{0}^{-1} l(y)$ of two different plateau components with exponents $\eta=2.5$ and 3.5 .

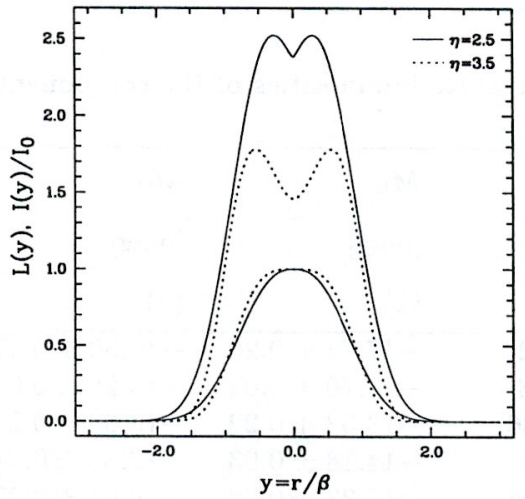

Fig. 10. Dimensionless luminosity density $L(y)$ versus dimensionless radius $y=r / \beta$ for plateau components with exponent $\eta=2.5$ (solid curve) and $\eta=3.5$ (dashed curve). The corresponding dimensionless plateau intensities $I\left(R^{*}\right) / I_{0}$ are shown in the lower part of the diagram

In both curves, the luminosity density reaches a local minimum at $y=0$. This central depression occurs for all plateau intensity distributions with $\eta>2$ and becomes more pronounced with increasing $\eta$ (Fig. 11). This does not mean that the luminosity density of the starburst component in a BCD actually decreases near the center, since a gaussian component with typically a much higher central luminosity density is invariably superimposed on the plateau component. This is illustrated for Mkn 86 in Fig. 12.

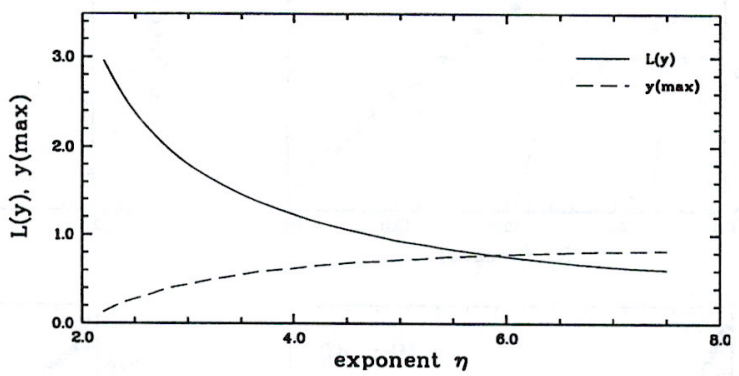

Fig. 11. Dimensionless luminosity density $L(y=0)$ (solid line) and radius $y(\max )$ at which $L(y)$ attains a maximum (dashed curve) as a function of plateau distribution exponent

\subsection{Deprojection of the exponential component}

Although young massive stars formed during the current or previous starbursts provide on average about half of the total $B$ light of a BCD (Paper II, Sect. 2.3), their dynamical influence is far less than that of the older LSB stellar population. Consequently $M_{*}(r)$ is primarily controlled by the characteristics of the underlying exponential component. To describe the SBPs of some BCDs (see Sect. 2.3.1) with a centrally flattened exponential light distribution, 


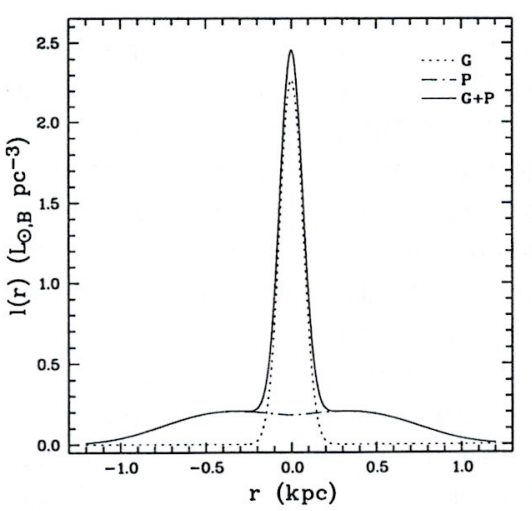

Fig. 12. Radial distributions of the luminosity densities of the gaussian component $l(r)_{\mathrm{G}}$ (dotted line), plateau component $l(r)_{\mathrm{P}}$ (dashed-dotted line) and starburst component $l(r)_{\mathrm{G}}+l(r)_{\mathrm{P}}$ (solid line) of $\mathrm{Mkn} 86$ in the $B$ band. The luminosity densities are in units of $L_{\odot, B} \mathrm{pc}^{-3}$. The exponent of the plateau is 2.9 , corresponding to a dimensionless luminosity density distribution $L(y)$ between the solid and dashed curves in Fig. 10

we propose a modification of the pure exponential law described by Eq. (3) as follows:

$$
I\left(R^{*}\right)=I_{0} \exp \left(-\frac{R^{*}}{\alpha}\right)\left\{1-q \exp \left(-P_{3}\left(R^{*}\right)\right)\right\}
$$

where $P_{3}\left(R^{*}\right)$ is

$$
P_{3}\left(R^{*}\right)=\left(\frac{R^{*}}{b \alpha}\right)^{3}+\left(\frac{R^{*}}{\alpha} \cdot \frac{1-q}{q}\right)
$$

The intensity distribution given by Eq. (22) depends near the center on $q=\Delta I / I_{0}$, where $I_{0}$ is the central intensity of a pure exponential law, and on the cutoff-radius $b \alpha$ within which flattening occurs. The function $P_{3}\left(R^{*}\right)$ vanishes as $R^{*}$ tends to 0 , and becomes much larger than unity for $R^{*}>b \alpha$. Thus, for $q>0$, Eq. (22) produces a centrally truncated exponential distribution similar to the type-V profiles discussed by Binggeli \& Cameron (1991), with $\mathrm{d} I / \mathrm{d} R^{*}=0$ for $R^{*}=0$ and a finite value for the central luminosity density.

The $(b, q)$ parameter space can be explored directly only when the central part of the underlying component is visible. Lacking such objects (there is always a central starburst component superimposed), $(b, q)$ parameter sets can be derived by requiring that the intensity increases smoothly and monotonically for $R^{*} \lesssim b \alpha$. After investigation of the $(b, q)$ parameter space, we adopt $b=3 q$. This is in qualitative agreement with $\overline{(b, q)}=(0.95 \pm 0.5,0.3 \pm 0.1)$ obtained by fitting Eq. (22) to 20 SBPs of dEs and dE,Ns taken from Binggeli \& Cameron (1991). Fig. 13 shows the intensity profiles resulting from Eq. (22) with the prescription $b=3 q$ for $q=0.1$ and 0.4 . For comparison, we also plot the distribution for $b=q$ and $q=0.5$. It is obvious that the latter distribution is not compatible with the observations.

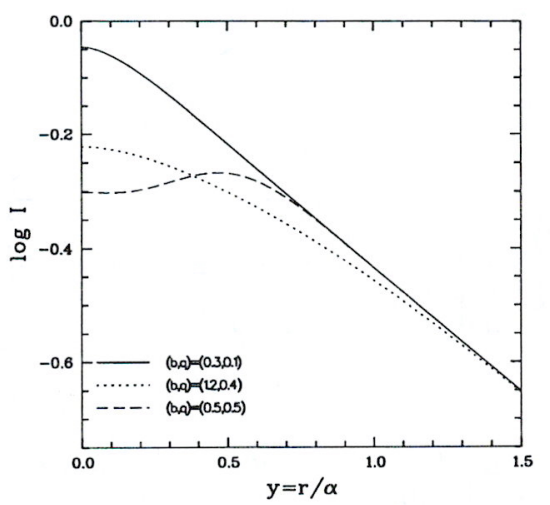

Fig. 13. Dimensionless intensity $I(y) / I_{0}$ for three $(b, q)$ sets in Eq. $(22)$ : $(0.3,0.1),(1.2,0.4)$ and $(0.5,0.5)$ near the center. Evidently, the third parameter set results in a non-monotonic intensity distribution for radii $\lesssim 0.6 \alpha$

Table 8. $\Delta M$ as a function of $(b, q)$

\begin{tabular}{lccc}
\hline$b$ & $q=\frac{\Delta I_{0}}{I_{0}}$ & $\begin{array}{c}\Delta M \\
\left(10^{-2} \mathrm{mag}\right)\end{array}$ & $\begin{array}{c}\Delta M_{3.4 \alpha} \\
\left(10^{-2} \mathrm{mag}\right)\end{array}$ \\
\hline 0.3 & 0.1 & 0.08 & 0.07 \\
0.6 & 0.2 & 0.61 & 0.71 \\
0.9 & 0.3 & 2.06 & 2.42 \\
1.2 & 0.4 & 4.96 & 5.83 \\
1.5 & 0.5 & 9.90 & 11.7 \\
\hline
\end{tabular}

The uncertainty in $(b, q)$ introduces in turn an uncertainty in the determination of the central luminosity density of the underlying component of BCDs. However the impact of the central flattening on the total luminosity of the exponential component is not large. This is evident from Eq. (11) which implies that the contribution of an intensity distribution $I\left(R^{*}\right)$ to the total luminosity is weighted with $R^{*}$. Column 3 of Table 8 lists the luminosity decrease $\Delta M$ if Eq. (22) is used to describe the underlying LSB component instead of a pure exponential, for different $(b, q)$ values.

Column 4 lists the same quantity as Col. 3, but gives the luminosity decrease within $E_{25}$, taken to be $3.4 \alpha$. This radius was derived from the average ratio $E_{25} / \alpha=3.4 \pm$ 0.14 in $B$ obtained from Tables 2 and 4 .

Figure 14 shows in a logarithmic representation the luminosity density corresponding to an intensity distribution of the form of Eq. (22) (solid and dashed curve respectively). A linear fit to the luminosity density in the 


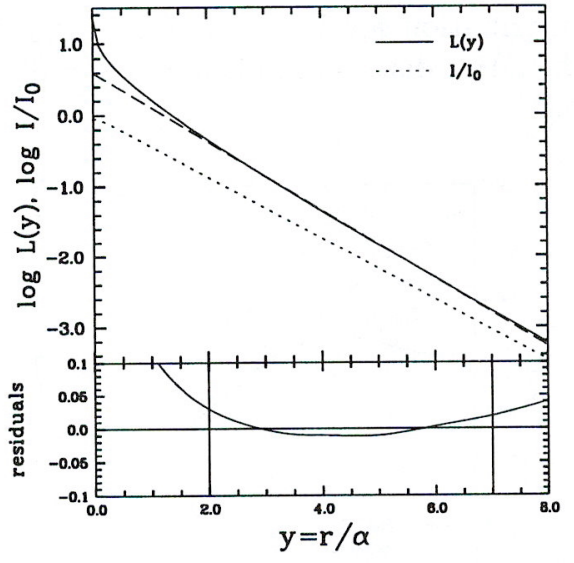

Fig. 14. Linear fit (dashed line) to the dimensionless luminosity density distribution $L(y)=\left(\alpha / I_{0}\right) l(y)$ (solid curve) corresponding to a modified exponential intensity distribution (Eq. 22) with $(b, q)=(0.3,0.1)$ (dotted line). $L(y)$ can be approximated in the range $2 \alpha \leq y \leq 7 \alpha$ by an exponential distribution with a scale length $\approx 0.9 \alpha$, slightly steeper than the projected intensity. The residuals between the fit and $L(y)$ are shown in the lower panel

range $2 \alpha \leq y \leq 7 \alpha$ yields the relation

$$
l(y) \approx \frac{4 I_{0}}{\alpha} \exp \left(-\frac{r}{0.9 \times \alpha}\right)
$$

between the luminosity density and the intensity. For the BCDs listed in Tables 2 and 4, we obtain a mean ratio $P_{25} / \alpha$ of $1.7 \pm 0.1$ in the $B$ band. Thus, Eq. (24) gives an approximation to the luminosity density of the underlying component of a $\mathrm{BCD}$ for radii $\gtrsim P_{25}$, where the underlying component becomes free from starburst light. Assuming a constant $\mathcal{M} / \mathcal{L}$ ratio one can then directly infer the intrinsic mass density distribution.

\subsection{Central luminosity densities of the three components}

In this section we give estimates for the $B$ central luminosity densities ${ }^{3}$ in units of $L_{\odot, B} \mathrm{pc}^{-3}$ for the galaxies listed in Table 9. Columns 3 and 4 list respectively the central luminosity densities $l_{\mathrm{P}, 0}$ of the plateau and $l_{\mathrm{G}, 0}$ of the gaussian component.

Clearly $l_{\mathrm{G}, 0}$ is strongly affected by seeing-dependent spatial resolution effects and is meant to be indicative only. High spatial resolution observations of star-forming regions in BCDs, as for instance He 2-10 (Conti \& Vacca 1994 ), indicate that a knot of $\sim 10 \mathrm{pc}$ in diameter may contain up to several thousands of massive stars. With the simplifying assumption that this young stellar population is distributed homogeneously within a spherical volume, we estimate a number density of $\lesssim 1 \mathrm{pc}^{-3}$. Since early-type stars have blue luminosities of several $10^{3} L_{\odot, B}$

\footnotetext{
${ }^{3}$ Throughout we adopt $M_{\odot, B}=5.54$.
}

we deduce a mean luminosity density within such a knot much higher than the $l_{\mathrm{G}, 0}$ values listed in Table 9.

With regard to the plateau component, we recall that the luminosity density reaches a local minimum at $R^{*}=0$ for exponents $\eta>2$. Because of seeing effects on the gaussian component and the large change in stellar populations within $P_{25}$, we avoid to estimate mass densities for the starburst component.

Column 2 yields the central luminosity density of the underlying component $l_{\mathrm{E}, 0}$, calculated by deprojection of Eq. (22) and assuming $(b, q)=(0.3,0.1)$. Whenever the data for the total and the HI masses are available, we estimate an approximate mass-to-luminosity ratio $\mathcal{M} / \mathcal{L}_{\mathrm{B}, \mathrm{E}} \simeq\left[M_{\mathrm{T}}-M(\mathrm{HI})\right] / L_{\mathrm{B}, \mathrm{E}}$ of the underlying component, assuming it to be constant (Col. 8). Estimates for the central stellar mass density $\rho_{\mathrm{E}, 0}$, in units of $M_{\odot} \mathrm{pc}^{-3}$, are listed in Col. 9.

\section{Color profiles}

As BCDs are known to be metal-deficient $\left(Z_{\odot} / 50 \lesssim Z \lesssim\right.$ $Z_{\odot} / 3$, Thuan 1991) their dust content is low, and color changes cannot be attributed to dust variation. The study of color gradients in BCDs therefore tells us about changes in stellar populations and/or metallicity as a function of radius. We use two different methods to derive the color profiles:

i) we directly subtract the R SBPs from the B SBPs. Before subtraction, the profile with the highest resolution is smoothed to the lower resolution of its counterpart. The results obtained in this way are shown Figs. 1e-6e by squares with error bars attached.

ii) While the first technique works well for $\mathrm{nE} B C D s$ with nuclear star formation, it may give misleading results for $\mathrm{iE} \mathrm{BCDs} \mathrm{in} \mathrm{some} \mathrm{cases.} \mathrm{In} \mathrm{nE}$ BCDs, the most luminous region in both $B$ and $R$ bands is also the bluest one, while in $\mathrm{iE} \mathrm{BCDs,} \mathrm{where} \mathrm{star} \mathrm{formation} \mathrm{is} \mathrm{spread} \mathrm{out} \mathrm{in}$ several knots, the most luminous region in a given band and the bluest region do not necessarily coincide. For example, a fading starburst knot containing a large number of intermediate-age stars from a previous starburst, can be the most luminous in $R$ (and $B$ ), whereas another knot in which the starburst has just begun can be the bluest but not necessarily the brightest one. Since the surface brightness decreases with radius, the first knot will determine the central surface brightness in both bands and hence the color index near the center, whereas the second knot will have little influence on the SBPs and the color profile. This would produce, on the average, redder colors in the region interior to the plateau radius than is really the case. To circumvent this problem, we applied to every color map a Fourier filter to suppress the noise and then used a method similar to that described in Sect. 2.2.1 (Eq. 2) to deduce the $B-R$ index as function of $R^{*}$, for photometric radii $\lesssim P_{25}$. The solid lines in Figs. 1e-6e illustrate the results obtained by this method. While for 
Table 9. Central luminosity densities of the components in the $B$ band

\begin{tabular}{|c|c|c|c|c|c|c|c|c|c|}
\hline Target & $\begin{array}{l}l_{\mathrm{E}, 0} \\
0.1 L_{\odot, B} \mathrm{pc}^{-3} \\
(2)\end{array}$ & $\begin{array}{l}l_{\mathrm{P}, 0} \\
0.1 L_{\odot, B} \mathrm{pc}^{-3} \\
(3)\end{array}$ & $\begin{array}{l}l_{\mathrm{G}, 0} \\
0.1 L_{\odot, B} \mathrm{pc}^{-3} \\
(4)\end{array}$ & $\begin{array}{l}M(\mathrm{HI})^{a} \\
10^{8} M_{\odot} \\
(5)\end{array}$ & $\begin{array}{l}M_{\mathrm{T}} \\
10^{8} M_{\odot} \\
(6)\end{array}$ & $\begin{array}{l}L_{\mathrm{B}, \mathrm{E}} \\
10^{8} L_{\odot, B} \\
(7)\end{array}$ & $\begin{array}{l}{\frac{\mathcal{M}}{\mathcal{L}_{\mathrm{B}, \mathrm{E}}}}^{b} \\
(8)\end{array}$ & $\begin{array}{l}\rho_{\mathrm{E}, 0} \\
M_{\odot} \mathrm{pc}^{-3} \\
(9)\end{array}$ & $\begin{array}{l}\mathrm{W} / \mathrm{N} \\
(10)\end{array}$ \\
\hline Haro 2 & $0.8 \pm 0.1$ & $0.2 \pm 0.03$ & $4.7 \pm 0.3$ & 4.6 & 15.0 & 10.1 & 1.03 & 0.09 & $\mathrm{~N}$ \\
\hline Haro 3 & $7.1 \pm 1.3$ & $7.8 \pm 2.7$ & $26.9 \pm 3.1$ & 5.4 & 12.0 & 6.8 & 0.97 & 0.7 & W \\
\hline \multirow[t]{2}{*}{ Mkn 5} & $18.2 \pm 1.4$ & $0.1 \pm 0.05$ & $18.9 \pm 1.4$ & 1.3 & 2.6 & 1.73 & 0.75 & 1.4 & $\mathrm{~N}$ \\
\hline & $13.9 \pm 1.6$ & $0.25 \pm 0.08$ & $19.17 \pm 1.44$ & 1.3 & 2.6 & 1.63 & 0.79 & 1.1 & W \\
\hline Mkn 7 & $3.2 \pm 0.2$ & - & - & - & - & 36.7 & - & - & $\mathrm{N}$ \\
\hline \multirow[t]{2}{*}{ Mkn 36} & $43.4 \pm 3.2$ & $2.9 \pm 0.5$ & $62.2 \pm 4.7$ & 0.19 & 0.25 & 0.31 & 0.19 & 0.84 & $\mathrm{~N}$ \\
\hline & $33.7 \pm 3.9$ & $2.1 \pm 0.2$ & $61.6 \pm 4.6$ & 0.19 & 0.25 & 0.29 & 0.21 & 0.70 & W \\
\hline \multirow[t]{2}{*}{ Mkn 49} & $11.6 \pm 0.4$ & $0.4 \pm 0.05$ & $30.0 \pm 2.3$ & 1.9 & 0.95 & 11.0 & - & - & $\mathrm{N}$ \\
\hline & $10.4 \pm 1.2$ & $0.7 \pm 0.2$ & $30.8 \pm 2.3$ & 1.9 & 0.95 & 10.6 & - & - & W \\
\hline \multirow[t]{2}{*}{ Mkn 86} & $2.4 \pm 0.4$ & $1.8 \pm 0.1$ & $22.7 \pm 2.9$ & 2.4 & 5.8 & 3.8 & 0.90 & 0.22 & $\mathrm{~N}$ \\
\hline & $2.8 \pm 0.6$ & $1.7 \pm 0.1$ & $22.7 \pm 1.7$ & 2.4 & 5.8 & 3.84 & 0.89 & 0.25 & W \\
\hline \multirow[t]{2}{*}{ Mkn 209} & $9.8 \pm 1.3$ & $8.7 \pm 5.6$ & $109 \pm 8$ & 0.426 & 1.82 & 0.30 & 4.65 & 4.53 & $\mathrm{~N}$ \\
\hline & $4.4 \pm 1.5$ & $3.2 \pm 1.3$ & $101 \pm 8$ & 0.426 & 1.82 & 0.26 & 5.36 & 2.37 & W \\
\hline \multirow[t]{2}{*}{ Mkn 297} & $0.5 \pm 0.03$ & $0.4 \pm 0.01$ & $22.3 \pm 0.2$ & 118 & - & 41.4 & - & - & $\mathrm{N}$ \\
\hline & $2.9 \pm 0.01$ & $0.2 \pm 0.01$ & $1.8 \pm 0.1$ & 118 & - & 89.5 & - & - & W \\
\hline \multirow[t]{2}{*}{ Mkn 400} & $12.0 \pm 0.4$ & $3.1 \pm 0.2$ & $58.0 \pm 4.4$ & 7.7 & 44.0 & 20.3 & 1.78 & 2.15 & $\mathrm{~N}$ \\
\hline & $11.4 \pm 0.7$ & $3.2 \pm 0.2$ & $58.5 \pm 4.4$ & 7.7 & 44.0 & 20.1 & 1.80 & 2.06 & W \\
\hline \multirow[t]{2}{*}{ Mkn 487} & $21.0 \pm 1.6$ & $13.7 \pm 5.2$ & $29.1 \pm 2.2$ & 0.49 & 0.49 & 0.52 & - & - & $\mathrm{N}$ \\
\hline & $14.2 \pm 0.7$ & $5.6 \pm 0.5$ & $36.4 \pm 2.7$ & 0.49 & 0.49 & 0.47 & - & - & W \\
\hline \multirow[t]{2}{*}{ I Zw 115} & $9.9 \pm 1.4$ & $0.2 \pm 0.01$ & - & 1.3 & 1.5 & 1.51 & 0.13 & 0.13 & $\mathrm{~N}$ \\
\hline & $10.1 \pm 1.7$ & $0.2 \pm 0.01$ & $0.7 \pm 0.1$ & 1.3 & 1.5 & 1.71 & 0.12 & 0.12 & W \\
\hline NGC 2915 & $8.1 \pm 0.6$ & $0.9 \pm 0.2$ & $36.2 \pm 2.7$ & 1.53 & 7.49 & 1.01 & 5.9 & 4.8 & $\mathrm{~N}$ \\
\hline
\end{tabular}

a: HI and total masses are from Thuan \& Martin (1981), except for Mkn 209 (Staveley-Smith et al. 1992), Mkn297 (Gordon \& Gottesman 1981) and NGC 2915 (Reif et al. 1982).

$b: \mathcal{M} / \mathcal{L}_{\mathrm{B}, \mathrm{E}}$ is estimated as $\simeq M_{\mathrm{T}}-M(\mathrm{HI}) / L_{\mathrm{B}, \mathrm{E}}$, where $L_{\mathrm{B}, \mathrm{E}}$ is the total luminosity of the underlying stellar component in $L_{\odot, B}$

most BCDs the results of the second method are in good agreement with those of the first method, they are very different for the non-BCD starburst galaxies Mkn 297 and I Zw 89.

From the center outwards, apart from local minima, the $B-R$ color first increases nearly linearly with radius. Mkn 209 (Fig. 3e) is the only BCD in our sample, which slightly deviates from this behavior, showing first a steep slope for small radii $\left(R^{*}<0.25 \mathrm{kpc}\right)$ and then a flattening for $0.25 \mathrm{kpc} \lesssim R^{*} \lesssim 0.6 \mathrm{kpc}$. The $B-R$ color then reaches a maximum corresponding to the reddest color and, frequently, for radii greater than the transition radius $R_{\mathrm{T}}$, there is a tendency for getting bluer again. However, the large photometric uncertainties in the outermost regions prevent a precise determination of this negative color gradient, and the possibility of the color being constant (cf. Loose \& Thuan 1986) cannot be ruled out.

A typical $B-R$ profile is that of Mkn 36 (Fig. 15) as derived by Diehl (1988) with the first method.

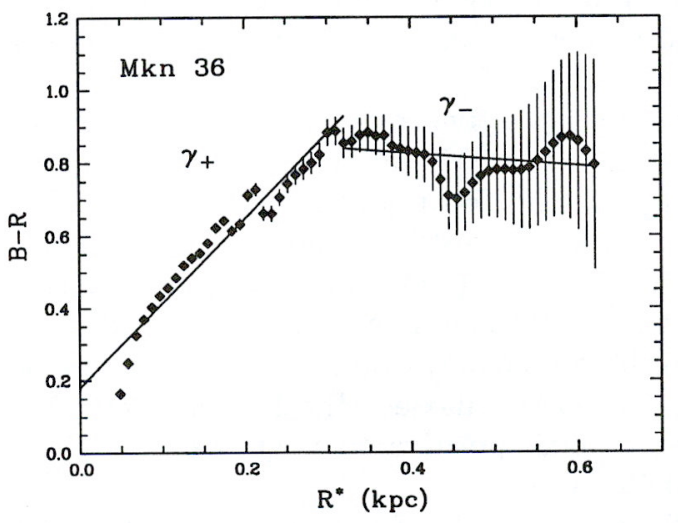

Fig. 15. $B-R$ profile of $\mathrm{Mkn} 36$ as derived by subtraction of the R SBP from the B SBP (Diehl 1988). The inner and outer color gradients, $\gamma_{+}$and $\gamma_{-}$respectively, are indicated

We can approximate the two parts of such a $B-R$ profile by the following linear fits:

$$
B-R=c_{1}+\gamma_{+} \times R^{*} \quad\left(R^{*} \leq R_{\mathrm{T}}\right)
$$




$$
B-R=c_{2}+\gamma_{-} \times R^{*} \quad\left(R^{*} \geq R_{\mathrm{T}}\right)
$$

where $R^{*}$ is the photometric radius in $\mathrm{kpc}, \gamma_{+}$and $\gamma_{-}$the color gradients of the inner and outer parts respectively, in mag $\mathrm{kpc}^{-1}, R_{\mathrm{T}}$ the transition radius in $\mathrm{kpc}$ and $c_{1}, c_{2}$ constants. The parameters entering in Eqs. (25) and (26) are also shown in Fig. 16 for the $B-R$ profile of $\mathrm{IZw} 115$ as determined with method I.

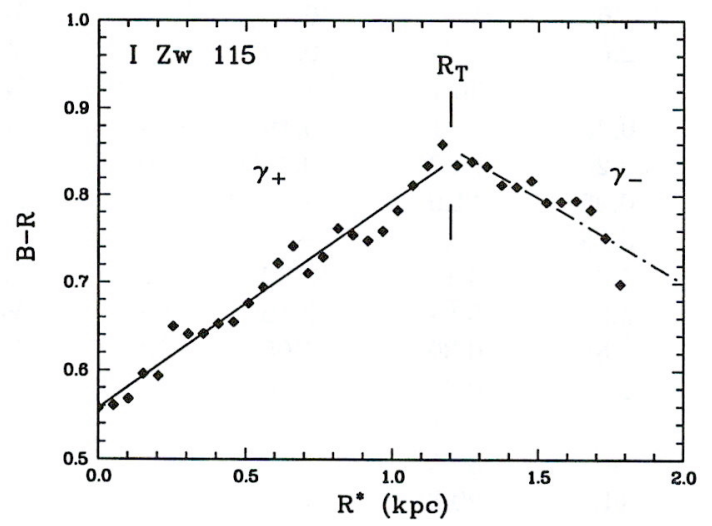

Fig. 16. $B-R$ profile of $\mathrm{IZw} 115$ derived according to method I. The positive and negative slopes of the profile $\gamma_{+}$ and $\gamma_{-}$and the transition radius $R_{\mathrm{T}}$ (Eqs. (25) and (26)) are indicated

Table 10 contains the parameters describing the $B-$ $R$ profiles of the galaxies under study for which both $B$ and $R$ data are available. Column 2 gives the extrapolated $B-R$ index $c_{1}$ at $R^{*}=0$, Cols. 3 and 4 the positive color gradients $\gamma_{+}^{\mathrm{I}}$ and $\gamma_{+}^{\mathrm{II}}$, as derived respectively from methods I and II, Col. 5 an approximative value for the transition radius $R_{\mathrm{T}}$, Col. 6 the ratio between $R_{\mathrm{T}}$ and the plateau radius $P_{25}$ and Col. 7 the negative color gradient $\gamma_{-}$.

The mean positive color gradient $\gamma_{+}$for BCDs as deduced from methods I and II is $0.8 \pm 0.2 \mathrm{mag} \mathrm{kpc}^{-1}$. The most compact BCDs in our sample, Mkn 36 and Mkn 209, also possess the largest color radients $\left(\gamma>1.5 \mathrm{mag} \mathrm{kpc}^{-1}\right)$. From Kunth et al. (1988) data, we found positive color gradients of $\sim 0.5 \mathrm{mag} \mathrm{kpc}-1$ in the BCDs Tol 2 and Tol 3 , comparable to the mean value derived here. As for the extended starburst galaxies Mkn 297 and I Zw 89, they exhibit very small positive color gradients of the order of $\gamma_{+} \lesssim 0.05 \mathrm{mag} \mathrm{kpc}^{-1}$.

Diffuse Hil emission from regions of active star formation contributes a considerable fraction of the light within the starburst component and thus $\mathrm{H} \alpha$ emission may, despite the large bandwidth of the $R$ filter, strongly influence the $B-R$ color index. Krüger et al. (1995) derived from model-calculations $B-R=1.1$ for a HII region with solar metallicity and $B-R=0.48$ for a Hil region with $Z_{\odot} / 20$. The BCDs in our sample have $0.0 \lesssim(B-R) \lesssim 0.88$ in their central parts. Thus only for the bluest BCDs in our sample, there is strong evidence that the dominant frac- tion of light is provided by young stars. In those cases the reddening in $B-R$ within $R_{\mathrm{T}}$ can be attributed to a change in stellar populations from very young $\left(\$ 10^{7} \mathrm{yr}\right)$ stars formed in the present burst of star-formation to older $\left(\lesssim 210^{9} \mathrm{yr}\right)$ stellar populations from previous starbursts which have diffused outwards. For $R^{*} \gtrsim P_{25}$, the light from the underlying older $(\gtrsim 10 \mathrm{Gyr})$ and redder stellar component begins to become important, dominating at $R^{*} \gtrsim R_{T}$. Negative color gradients beyond $R_{T}$ may be attributed to a decrease in metallicity or in mean stellar age (cf. Vader et al. 1988). The slope $\gamma_{-}$ranges between 0.0 and -0.3 (Table 10, Col. 7), with an average value of $\overline{\gamma_{-}} \simeq-0.14 \pm 0.04 \mathrm{mag} \mathrm{kpc}^{-1}$. These negative color gradients are reminiscent of the ones found in early-type dwarf galaxies (Vigroux et al. 1988; Vader et al. 1988).

\section{Conclusions}

The main conclusions of this work are as follows:

1. BCDs form a heterogeneous class of galaxies as regard their morphology and surface brightness profiles (SBPs). However, a closer look into the $\mathrm{nE}$ and iE subclasses which contain the vast majority of BCDs, reveals a connection between morphology and the light distribution:

a. BCDs of the nE type, i.e. those with nuclear star-forming activity, show SBPs which are frequently well fitted by a de Vaucouleurs or a power law.

b. By contrast BCDs of the $\mathrm{iE}$ type, with multiple knots of star formation spread over a large fraction of their surface, exhibit a plateau feature at intermediate intensity levels in their SBPs. Such SBPs resemble those of S0 galaxies and cannot be described by a single fitting law.

While the above two types of BCDs differ at high intensity levels, they appear fairly similar at surface brightness levels fainter than $\sim 24 \mathrm{mag} \operatorname{arcsec}^{-2}$ in $\mathrm{B}$, where the more extended faint stellar population dominates the light. This underlying LSB component has mostly elliptical isophotes and is characterized by redder colors, suggesting an older stellar population formed prior to the present starburst.

2. We have applied a 3-component decomposition scheme to the SBPs consisting of:

(a) A gaussian component which describes the intensity distribution of the most luminous knot in the starburst region.

(b) A plateau component which is most prominent at intermediate intensity levels in $\mathrm{iE} \mathrm{BCDs}$ and less important in $\mathrm{nE} \mathrm{BCDs.} \mathrm{Its} \mathrm{intensity} \mathrm{can} \mathrm{be}$ described by a modified exponential distribution characterized by 3 parameters: the central intensity, the scale length and the exponent $\eta$ which controls the curvature of the fit. 
Table 10. Color gradients

\begin{tabular}{lccccccc}
\hline Name & $\begin{array}{c}c_{1} \\
{[\mathrm{mag}]}\end{array}$ & $\begin{array}{c}\gamma_{+}^{\mathrm{I}} \\
{\left[\frac{\mathrm{mag}}{\mathrm{kpc}}\right]}\end{array}$ & $\begin{array}{c}\gamma_{+}^{\mathrm{II}} \\
{\left[\frac{\mathrm{mag}}{\mathrm{kpc}}\right]}\end{array}$ & $\begin{array}{c}R_{\mathrm{T}} \\
{[\mathrm{kpc}]}\end{array}$ & $R_{\mathrm{T}} / P_{25}$ & $\begin{array}{c}\gamma_{-} \\
{\left[\frac{\mathrm{mag}}{\mathrm{kpc}}\right]}\end{array}$ & $\mathrm{N} / \mathrm{W}$ \\
$(1)$ & $(2)$ & $(3)$ & $(4)$ & $(5)$ & $(6)$ & $(7)$ & $(8)$ \\
& & & & & & & \\
\hline Haro 2 & 0.53 & 0.32 & - & 1.8 & 1.0 & -0.32 & $\mathrm{~N}$ \\
Haro 3 & 0.67 & 0.26 & 0.34 & 2.5 & 2.1 & -0.11 & $\mathrm{~W}$ \\
Mkn 36 & 0.18 & 2.34 & - & 0.32 & $1.1 / 1.1$ & -0.17 & $\mathrm{~N} / \mathrm{W}$ \\
Mkn 86 & 0.90 & 0.29 & 0.35 & 1.25 & $1.0 / 1.0$ & -0.21 & $\mathrm{~N} / \mathrm{W}$ \\
Mkn 209 & 0.18 & 1.55 & 1.60 & 0.65 & $1.9 / 1.7$ & $\sim 0.0$ & $\mathrm{~N}$ \\
Mkn 297 & 0.60 & - & 0.043 & $\sim 8.4$ & $1.2 / 1.3$ & -0.1 & $\mathrm{~N} / \mathrm{W}(a)$ \\
I Zw 89 & 0.98 & - & 0.05 & $\sim 4.9$ & - & -0.07 & $\mathrm{~N} / \mathrm{W}(a)$ \\
IZw 115 & 0.56 & 0.24 & 0.29 & 1.2 & $2.5 / 2.3$ & -0.20 & $\mathrm{~N} / \mathrm{W}$ \\
NGC2915 & 0.98 & 1.1 & - & $\sim 1.0$ & $\sim 2.3$ & $\simeq 0.0$ & $\mathrm{~N}$ \\
\hline
\end{tabular}

$a$ : The extrapolated color index at $R^{*}=0$ and the approximative color index at $P_{25}$ are obtained with method II.

(c) An exponential component which describes the intensity of the older LSB stellar component. The scale length and central intensity of this component are determined from the fit to the outer regions where the light contribution from young $\mathrm{OB}$ star associations and nebular emission is negligible. As the SBPs of some BCDs (for instance I Zw 115) suggest a flattening of the exponential component near the center, we discuss also a modified exponential fitting law to account for that behavior.

The decomposition scheme yields the following physical quantities: a) the central surface brightness and scale length of the underlying LSB stellar component; b) the extents of the underlying galaxy $E_{25}$ and of the starburst component $P_{25}$ determined as the radii at $25 \mathrm{mag} \operatorname{arcsec}^{-2}$ of the exponential and the plateau components, respectively. Both quantities are typically much larger than the resolution limit of ground-based observations and are thus not seeing-dependent. c) the concentration index $C I$ (Eq. 10) which measures the surface of the star-forming region relative to the total surface of a $\mathrm{BCD}$, and, by definition, increases from $\mathrm{iE}$ to $\mathrm{nE}$ BCDs. d) the luminosity of the starburst component equal to the sum of the luminosities of gaussian and plateau components and e) that of the underlying galaxy, described by the exponential component.

3. For small radii, $B-R$ color profiles of most BCDs show a reddening, with an average linear color gradient $\gamma_{+} \sim 0.8 \mathrm{mag} \mathrm{kpc}^{-1}$. There appears to be a relation between the compactness of the galaxy and the steepness of the color gradient. The most compact members in our sample, Mkn 209 and Mkn 36, exhibit strong color gradients $\left(\gamma_{+}>1.5 \mathrm{mag} \mathrm{kpc}^{-1}\right)$, whereas the color gradients of the two extended non-BCD galax- ies, I Zw 89 and Mkn 297, included in this study for comparison, are much smaller $\left(\lesssim 0.05 \mathrm{mag} \mathrm{kpc}^{-1}\right)$. Beyond the transition radius $R_{\mathrm{T}}$, the photometric uncertainties prevent a decisive determination of the color gradient. There is, however, a general tendency for a blueing with increasing radius, perhaps due to a decrease in metallicity and/or mean stellar age outwards.

4. Assuming spherical symmetry, the three luminosity components resulting from the decomposition have been deprojected and intrinsic luminosity densities have been derived.

In a subsequent paper (Papaderos et al. 1996), we shall use the above data to investigate correlations between different physical parameters and gain insight into star-forming processes in BCDs. We shall also compare the structural parameters derived here for BCDs to those of other types of dwarf galaxies such as dwarf irregulars and dwarf ellipticals in view of possible evolutionary connections between them.

Acknowledgements. Research by P. Papaderos, H.-H. Loose and K.J. Fricke has been supported in part by BMFT Verbundforschung Astronomie grant 05 5G042A and Deutsche Agentur für Raumfahrtangelegenheiten (DARA) GmbH grant 50 OR 9407 6. T.X. Thuan acknowledges the partial financial support of the Commissariat à l'Energie Atomique and of a Visiting Professorship at the Université de Paris 7. T.X. Thuan would like to thank the hospitality of L. Vigroux in the Service d'Astrophysique at Saclay and T.X. Thuan and P. Papaderos thank C. Balkowski for welcoming them in the Departement d'Astronomie Extragalactique et Cosmologie at the Observatoire de Meudon. We thank the referee, B. Binggeli, for numerous comments which have helped to clarify various aspects of this work. This research has made use of the 
NASA/IPAC extragalactic database (NED) which is operated by the Jet Propulsion Laboratory, Caltech, under contract with the National Aeronautics and Space Administration.

\section{References}

Alloin D., Duflot R., 1979, A\&A 78, L5

Barnes J.E., Hernquist L.E., 1991, ApJ 370, 65

Binggeli B., Sandage A., Tarenghi M., 1984, AJ 89, 64

Binggeli B., Cameron L.M., 1991, A\&A 252, 27

Binggeli B., Cameron L.M., 1993, A\&AS 98, 297

Bothun G.D., Mould J.R., Caldwell N., MacGillivray H.T., 1986, AJ 92, 1007

Bothun G.D., Halpern J.P., Lonsdale C.J., Impey C., Schmitz M., 1989, ApJS 70, 271

Bothun G.D., Impey C.D., Malin D.F., 1991, ApJ 376, 404

Campbell A.W., Terlevich R., 1984, MNRAS 211, 15

Campos-Aguilar A., Moles M., 1991, A\&A 241, 358

Cellone S.A., Forte J.C., Geisler D., 1994, ApJS 93, 397

Christian C.A., Adams M., Barnes J.V., etal., 1985, PASP 97, 363

Conti P.S., Vacca W.D., 1994, ApJL 423, L97

Davies J.I., Phillipps S., 1988, MNRAS 233, 553

Deharveng J.-M., Albrecht R., Barbieri C., et al., 1994, A\&A 288, 413

De Young D.S., Gallagher J.S. III, 1990, ApJ 356, L15

De Young D., Heckman T.M., 1994, ApJ 431, 598

Diehl M., 1988, Diplom Thesis, Göttingen

Doi M., Fukugita M., Okamura S., 1995, ApJS 97, 59

Drinkwater M., Hardy E., 1991, AJ 101, 94

Fanelli M.N., O'Connell R.W., Thuan T.X., 1988, ApJ 334, 665

Freeman K.C., 1970, ApJ 160, 811

Freitag V., 1992, Diplom Thesis, Göttingen

Gordon D., Gottesman S.T., 1981, AJ 86, 161

Hecquet J., Coupinot G., Maucherat A.J., 1987, A\&A 183, 13

Heeschen D.S., Heidmann J., Yin Q.F., 1983, ApJ 267, L73

Hubble E., 1930, ApJ 71, 231

Izotov Y., Guseva N., Lipovetsky V., Kniazev A., 1993, in "Panchromatic view of galaxies". In: Hensler G., Theis Ch., Gallagher J. (eds.). Paris: Editions Frontières, p. 192

James P.A., 1991, MNRAS 250, 544

James P.A., 1994, MNRAS 269, 176

King I.R., 1966, AJ 71, 64

Kormendy J., 1977, ApJ 218, 333

Krüger H., Fritze-v. Alvensleben U., Loose H.-H., Fricke K.J., 1991, A\&A 242, 343

Krüger H., Fritze-v. Alvensleben U., Loose H.-H., 1995, A\&A 303,41

Kunth D., Sargent W.L.W., 1986, ApJ 300, 496

Kunth D., Maurogordato S., Vigroux L., 1988, A\&A 204, 10

Lequeux J., Peimbert M., Rayo J.F., Serrano A., TorresPeimbert S., 1979, A\&A 80, 155

Lequeux J., Kunth D., Mas-Hesse J.M., Sargent W.L.W., 1995, A\&A 301, 18

Lindner U., Einasto M., Einasto J., et al., 1996, A\&A (in press)

Loose H.-H., Thuan T.X., 1985, in "Star - Forming Dwarf Galaxies and Related Objects". In: Kunth D., Thuan T.X.,
Van T.T. (eds.). Paris: Editions Frontières, p. 73

Loose H.-H., Thuan T.X., 1986, ApJ 309, 59

Marconi G., Matteucci F., Tosi M., 1994, MNRAS 270, 35

Marlowe A.T., Heckman T.M., Wyse R.F.G., Schommer R., 1995, ApJ 438, 563

Marquardt D.W., 1963, J. Soc. Ind. Appl. Math. 11, 431

Matteucci F., Chiosi C., 1983, A\&A 123, 121

McGaugh S.S., Bothun G.D., 1994, AJ 107, 530

Meurer G.R., Freeman K.C., Dopita M.A., Cacciari C., 1992, AJ 103,60

Meurer G.R., Mackie G., Carignan C., 1994, AJ 107, 2021

Okamura S., Kodaira K., Watanake M., 1980, ApJ 280, 7

Papaderos P., Fricke K.J., Thuan T.X., Loose H.-H., 1994, A\&A 291, L13

Papaderos P., Loose H.-H., Fricke K.J., Thuan T.X., 1996, A\&A (in press) (Paper II)

Pilyugin L.S., 1994, A\&A 287, 387

Pustil'nik S.A., Ugryumov A.V., Lipovetsky V.A., Thuan T.X., Guseva N.G., 1995, ApJ 443, 499

Reif K., Mebold U., Goss W.M., van Woerden H., Siegman B., 1982, A\&AS 50, 451

Rosenberg J.L., Salzer J.J., Moody J.W., 1994, AJ 108, 1557

Reynolds J.H., 1913, MNRAS 74, 132

Roy J.-R., Aubé M., McCall M.L., Dufour R.J., 1992, ApJ 386,498

Roy J.-R., Kunth D., 1995, A\&A 294, 432

Rönnback J., Bergvall N., 1994, A\&AS 108, 193

Salzer J.J., 1989, ApJ 347, 152

Sargent W.L.W., Searle L., 1968, ApJ 152, 443

Sargent W.L.W., Filippenko A.V., 1991, AJ 102, 107

Searle L., Sargent W.L.W., 1972, ApJ 173, 25

Searle L., Sargent W.L.W., Bagnuolo W.G., 1973, ApJ 179, 427

Staveley-Smith L., Davies R.D., Kinman T.D., 1992, MNRAS 258,334

Taniguchi Y., Noguchi M., 1991, AJ 101, 1601

Telles E., Terlevich R., 1995, MNRAS 275, 1

Thuan T.X., Martin G.E., 1981, ApJ 247, 823

Thuan T.X., 1983, ApJ 268, 667

Thuan T.X., 1985, ApJ 299, 881

Thuan T.X., 1991, in "Massive Stars in Starbursts". In: Leitherer C., Walborn N.R., Heckman T.M., Norman C.A. (eds.). Cambridge: Cambridge University Press, p. 183

Thuan T.X., Izotov Y.I., Lipovetsky V.A., Pustil'nik S.A, 1994, in ESO/OHP workshop "Dwarf Galaxies". In: Meylan G., Prugniel P. (eds.). Garching: ESO, 421

Vader J.P., 1986, ApJ 305, 669

Vader J.P., Vigroux L., Lachièze-Rey M., Souviron J., 1988, A\&A 203, 217

Vader J.P., Chaboyer B., 1994, AJ 108, 1209

Viallefond F., Thuan T.X., 1983, ApJ 269, 444

Vigroux L., Stasińska G., Comte G., 1987, A\&A 172, 15

Vigroux L., Souviron J., Lachièze-Rey M., Vader J.P., 1988, A\&AS 73, 1

Vorontsov-Velyaminov B.A., 1977, A\&AS 28, 3

Young C.K., Currie M.J., 1994, MNRAS 268, L11

Young C.K., Currie M.J., 1995, MNRAS 273, 1141 